\title{
Cyclic Symmetry Finite Element Forced Response Analysis of a Distortion-Tolerant Fan with Boundary Layer Ingestion
}

\author{
J.B. Min, T.S.R. Reddy ${ }^{\dagger}$, M.A. Bakhle \\ and \\ R.M. Coroneos, G.L. Stefko \\ A.J. Provenza, K.P. Duffy ${ }^{\dagger}$ \\ NASA Glenn Research Center, Cleveland, Ohio \\ University of Toledo, Toledo, Ohio
}

\begin{abstract}
Accurate prediction of the blade vibration stress is required to determine overall durability of fan blade design under Boundary Layer Ingestion (BLI) distorted flow environments. Traditional single blade modeling technique is incapable of representing accurate modeling for the entire rotor blade system subject to complex dynamic loading behaviors and vibrations in distorted flow conditions. A particular objective of our work was to develop a high-fidelity full-rotor aeromechanics analysis capability for a system subjected to a distorted inlet flow by applying cyclic symmetry finite element modeling methodology. This reduction modeling method allows computationally very efficient analysis using a small periodic section of the full rotor blade system. Experimental testing by the use of the 8-foot by 6-foot Supersonic Wind Tunnel Test facility at NASA Glenn Research Center was also carried out for the system designated as the Boundary Layer Ingesting Inlet/Distortion-Tolerant Fan (BLI'DTF) technology development. The results obtained from the present numerical modeling technique were evaluated with those of the wind tunnel experimental test, toward establishing a computationally efficient aeromechanics analysis modeling tool facilitating for analyses of the full rotor blade systems subjected to a distorted inlet flow conditions. Fairly good correlations were achieved hence our computational modeling techniques were fully demonstrated. The analysis result showed that the safety margin requirement set in the BLI'DTF fan blade design provided a sufficient margin with respect to the operating speed range.
\end{abstract}

\section{Introduction}

$\mathrm{T}$ HE concept of relocating the engines from a podded position on the wing or fuselage and embedding them within the airframe ${ }^{[1]}$ (Figure 1) can improve vehicle fuel burn, weight, noise, and emissions (through reduced fuel burn and engine cycle improvements). This Hybrid-Wing-Body (HWB) architecture is particularly suited to embedded engines, as balance requirements already place them near the aft of the airframe. ${ }^{[2]}$ The embedded engines on a HWB can be integrated into the fuselage and ducted to exhaust through the rear of the vehicle. A main feature of this proposed boundary layer ingested inlet distortion-tolerant fan aircraft is the embedded aft-mounted engines which ingest part of the fuselage boundary layer air flow to reduce fuel burn. This embedded aft engines may also provide reduced susceptibility to bird strike since engines are partly hidden head-on especially at take-off, and possibly enabling to reduce the engine noise to the ground from positioning the engines above the airframe. The National Aeronautics and Space Administration (NASA) and United Technologies Research Center (UTRC) with contributions from Virginia Polytechnic and State University (Virginia Tech) have developed a BLI propulsor to significantly reduce the fuel consumption. Accordingly, a high-efficiency Boundary Layer Ingestion (BLI) flow Distortion-Tolerant Fan (DTF) blade rotor system has been studied as part of the Boundary Layer Ingesting Inlet/Distortion-Tolerant Fan (BLI²DTF) Project. 
Inlet airflow distortion ingestion into a fan gives rise to additional aero-mechanical vibration and a decrease in stability margin. An embedded BLI propulsion system inherently involves a persistent and severe inlet distortion reaching the fan at most operating conditions, resulting in high dynamic stresses due to aeroelastic forced responses and the possibility of flutter. If not adequately addressed by the propulsor design, these aeromechanics phenomena could cause fan structural failure due to high cycle fatigue (HCF) or unstable self-excited failure, respectively. Numerical simulation modelling for this dynamically interconnected problem is a challenging problem.

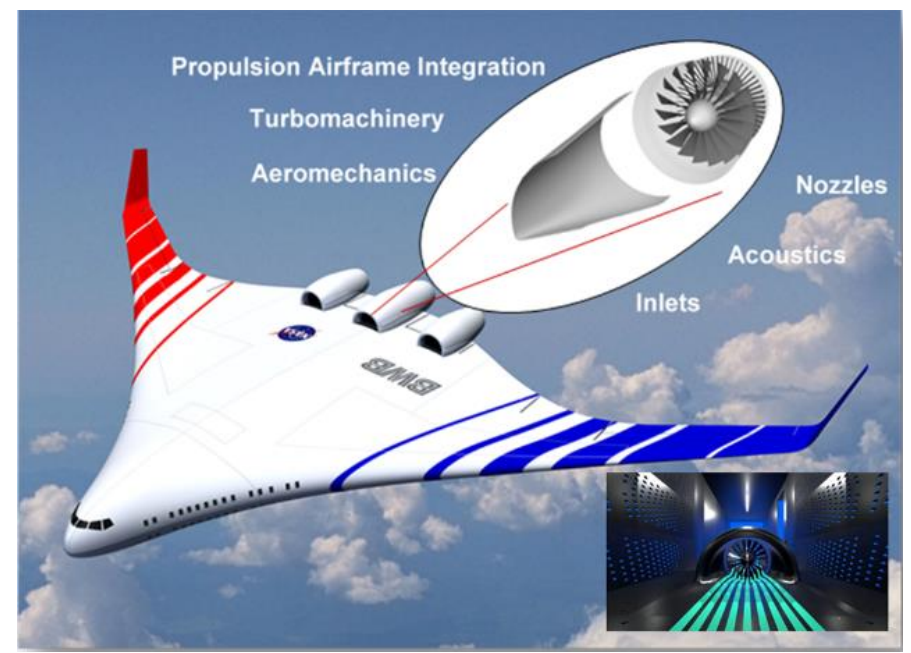

Figure 1: Conceptual aircraft design and boundary layer ingestion inlet distortion-tolerant rotor-fan system (inset at the bottom: BLI'DTF fan in the 8'x6' Supersonic Wind Tunnel test at NASA GRC)

Aero-mechanical vibration stresses in fan blades due to inlet airflow distortion is of particular interest in this study since the increase of stress in a blade can lead to its premature failure. Blade failure is usually occurring within short time by unstable self-excited (flutter) overload and high cycle fatigue (HCF) dynamic load. Turbomachinery blade vibration stress problem has been reported as the single cause to termination in new engine development effort. "While over 90 percent of the potential HCF problems are uncovered during development testing of a new engine, the remaining few account for nearly 30 percent of the total development cost and are responsible for over 25 percent of all engine distress events." $[3]$

One of the main research objectives of the BLI ${ }^{2}$ DTF Project was to develop and apply aeromechanics analysis tools to predict the effects of unique BLI distortion on "designed to be distortion-tolerant" fan system. ${ }^{[4,5]}$ Traditional single blade modeling techniques are incapable of accurately modeling the entire rotor blade system due to complex dynamic loading behaviors and vibrations in distorted flow conditions. Thus, the present aeromechanics analysis on BLI $^{2}$ DTF fan rotor system had four specific objectives: (1) establish a model based approach that enables a highfidelity full-rotor aeromechanics analysis capability for performance, safety, and computational efficiency in design analysis of a fan rotor system subjected to a distorted inlet flow, (2) complete forced response analyses to determine dynamic stresses in DTF fan blade due to continual operation in a distorted flow resulting from BLI using cyclic symmetry modeling techniques, (3) assess the concerns of fatigue problem using Goodman Diagram approach by examining predicted stress values regarding the HCF behavior due to blade static and vibration dynamic stresses, and (4) provide guidance on the strain measurement by determining optimal strain gauge locations on the blades towards a cost-effective experimental test measurement from overall and/or directional strain characteristics predicted due to a loading condition aforementioned. This latest high-fidelity modeling capability enables the analysis of arbitrary inlet distortion patterns that can be specified through variations of flow properties, together with prescribed characteristics of DTF fan blade vibrations. The present analysis approach will ensure operability of DTF fan blades in the embedded propulsion system of HWB aircraft.

\section{Cyclic symmetry finite element modeling technique}

Understanding complex mechanical and aerodynamic interaction of turbomachine blading in this distorted flow environment has become topmost to the success of $\mathrm{BLI}^{2} \mathrm{DTF}$ fan design and durability. As mentioned above, typical single blade analysis models widely employed in the turbomachinery research community are incapable of representing the design for both understanding the harmonic modal blade dynamic behaviors and accurately predicting 
corresponding forced response information of the blades subjected to complex distorted flow dynamic loading and vibrations. Nevertheless, analyzing this type of the problems by modeling a full 360 degree rotor blades structural system is not computationally efficient and practical, especially in large size systems. With addressing this issue in the present DTF fan analysis, reduction modeling techniques were essential and a novel reduction modeling capability was developed for fan distortion analysis to model non-axisymmetric large turbomachinery BLI ${ }^{2} \mathrm{DTF}$ rotor blades system. To achieve this capability, cyclic symmetry analysis modeling formulations were incorporated with the Ansys finite element (FE) computer program ${ }^{[6]}$ to analyze the $\mathrm{BLI}^{2} \mathrm{DTF}$ forced responses.

Cyclic symmetry is a type of symmetry in which identical sectors are attached in a circular pattern about a rotation axis. It is assumed that in each individual sector's local coordinate system the stiffness and mass matrices for all of the sectors are identical. The reduction of degrees-of-freedom is accomplished at the sector level. The compatibility of sector interfaces generates a reduced set of equations of the complete structure. A key element of cyclic symmetry method is to determine the cyclic symmetry transformation. That is, the solution of the complete structure can be reduced to the solution of the cyclic symmetric substructures. Once the solution for the substructure is determined, the solution of the complete structure is given by the cyclic symmetry transformation. The general procedure for the implementation of this method is given in reference 7.

The sector geometry of a BLI ${ }^{2} \mathrm{DTF}$ fan rotor system was meshed ${ }^{[8]}$ with higher order 20-node hexahedral elements for the airfoil region. Higher order 10-node tetrahedral elements for the airfoil platform and the disk part were more densely meshed for better prediction of stress results. This sector model represents a cyclic symmetric sector of a whole DTF fan rotor system, shown in Figure 2.

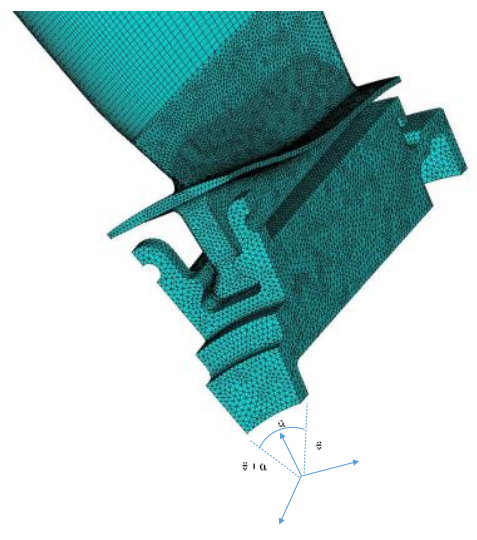

(a)

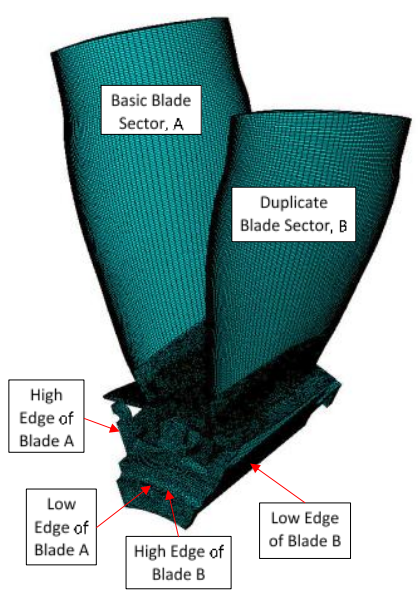

(b)

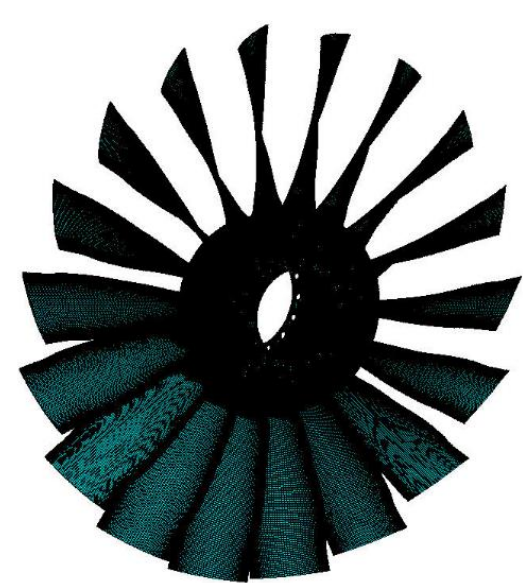

(c)

Figure 2: a) FE mesh of a cyclically symmetric "base sector" model of BLI'DTF (close-up), b) description of cyclic symmetry interface boundaries, c) fully expanded mesh by cyclic symmetry formulation

Eighteen blades were placed symmetrically around the disk, so a 20 degree sector was used to produce a symmetric sector. The airfoil itself was meshed with 4 hexahedral elements through the thickness in order to properly capture the bending stiffness. The resulting mesh of a sector geometry having 557,554 elements and 992,548 nodes is shown in Figure 2 which requires to solve 5,912,580 equations for a particular engine operating condition. It was anticipated to be able to analyze this extremely large model by solving the equations only for a smaller sized sector model without losing the computational accuracy rather than analyzing the entire 360 degree rotor blade model requiring unrealistic computing time and hardware capacity.

The cyclic sector model has two edges that align along the surfaces of cyclic symmetry (Fig. 2, (a)) in angles. The edge having the algebraically lower angle, $\theta$, in the cylindrical coordinate system is called the low edge and the one having the higher angle, $\theta+\alpha$, is called the high edge. The angle, $\alpha$, between the two successive surfaces of cyclic symmetry is called the sector angle. The architecture of the cyclic symmetry solution process depends upon how the compatibility and equilibrium conditions of the cyclic sector are enforced in the matrix-solution process using the duplicate sector method. ${ }^{[7]}$

The principal features of this cyclic symmetry modeling method are briefly described below. Along with the notations illustrated in Figure 2(b), all loading, boundary conditions, and coupling and constraint equations being 
present on the basic sector $(A)$ are applied to the duplicate sector $(B)$. Constraint relationships (Eqn. (1)) can be defined to relate the lower $(\theta=0)$ and higher $(\theta=\alpha)$ angle edges of the basic sector to allow calculations related to a given number of harmonic indices $(k)$ defined below. Essentially, the basic sector is duplicated in the analysis steps to satisfy the required constraint relationships and to obtain nodal displacements.

$$
\left\{\begin{array}{l}
u_{A}^{\prime} \\
u_{B}^{\prime}
\end{array}\right\}=\left[\begin{array}{cc}
\cos k \alpha & \sin k \alpha \\
-\sin k \alpha & \cos k \alpha
\end{array}\right]\left\{\begin{array}{l}
u_{A} \\
u_{B}
\end{array}\right\}
$$

where:

$u_{A}, u_{B}=$ calculated displacement on lower angle side of basic and duplicated sectors (A and

$\mathrm{B}$, respectively)

$u_{A}^{\prime}, u_{B}^{\prime}=$ displacements on higher angle side of basic and duplicated sectors (A and B, respectively) determined from constraint relationships

$$
\begin{aligned}
& k=\text { harmonic index }=0,1,2 \ldots\left\{\begin{array}{l}
\frac{N}{2} \text { if } N \text { is even } \\
\frac{N-1}{2} \text { if } N \text { is odd }
\end{array}\right. \\
& \alpha=2 \pi / N=\text { sector angle } \\
& N=\text { total number of sectors in } 360 \text { degree }(N=18)
\end{aligned}
$$

The cyclic symmetric solution sequences consist of three basic steps. The first step transforms applied loads to cyclic symmetric components using finite Fourier theory and enforces cyclic symmetry constraint equations (Eqn. (1)) for each harmonic index (i.e. nodal diameter) $(k=0,1, \ldots, N / 2)$. At the solution stage of a cyclic symmetry analysis, the cyclic symmetry compatibility conditions are enforced for each harmonic index solution via coupling and/or constraint equations (CEs) connecting the nodes on the low- and high-edge components on the basic and duplicate sectors. As stated earlier, the cyclic symmetry solution process depends upon how the compatibility and equilibrium conditions of the cyclic sector are enforced in the matrix-solution process. The solution stage performs each harmonic index solution as a separate load step where the real (basic sector) and imaginary (duplicate sector) parts of the solution are calculated, as expressed below in Eqn. (2) - Eqn. (5). The Fourier transformation from physical components, $u$, to the different harmonic index components, $\bar{u}$, is given by,

For harmonic index, $\mathrm{k}=0$ (symmetric mode),

$$
\bar{u}_{k=0}=\frac{1}{N} \sum_{n=1}^{N} u_{n}
$$

For harmonic index, $0<\mathrm{k}<\mathrm{N} / 2$ (mix mode), basic sector:

$$
\left(\bar{u}_{k}\right)_{A}=\frac{2}{N} \sum_{n=1}^{N} u_{n} \cos (n-1) k \alpha
$$

duplicate sector:

$$
\left(\bar{u}_{k}\right)_{B}=\frac{2}{N} \sum_{n=1}^{N} u_{n} \sin (n-1) k \alpha
$$

For $\mathrm{N}$ even only, $\mathrm{k}=\mathrm{N} / 2$ (anti-symmetric mode):

$$
\bar{u}_{k=N / 2}=\frac{1}{N} \sum_{n=1}^{N}(-1)^{(n-1)} u_{n}
$$

where:

$$
\begin{aligned}
& u=\text { displacements }(\text { or any physical component, such as, forces, pressure loads, temperatures, and inertial loads } \\
& \bar{u}=\text { cyclic displacements (or any cyclic symmetric component) } \\
& n=\text { sector number, varies from } 1 \text { to } N
\end{aligned}
$$

In the second step, any applied load on the full 360 degree fan model is treated through a Fourier transformation process above and applied on to the cyclic basic sector. For each value of harmonic index, $k$, the procedure solves the corresponding equation. In the third step, the responses are then combined to get the complete response of the full fan structure via the Fourier expansion given by Eqn. (6). That is, using the cyclic symmetry solution of the basic and duplicate sectors connecting low and high edges of basic and duplicate sectors combines the solutions from the two sectors by performing computations on the selected load step to combine the results of the two sectors. Note that only a harmonic index zero solution $(k=0)$ is valid for a static solution with cyclic loading. 
The transformation to physical components, $u$, from the cyclic symmetry, $\bar{u}$, components is recovered by this equation,

$$
u_{n}=\bar{u}_{k=0}+\sum_{k=1}^{N}\left[\left(\bar{u}_{k}\right)_{A} \cos (n-1) k \alpha+\left(\bar{u}_{k}\right)_{B} \sin (n-1) k \alpha\right]+(-1)^{(n-1)} \bar{u}_{k=N / 2}
$$

The last term $(-1)^{(n-1)} \bar{u}_{k=N / 2}$ exists only for a case of $\mathrm{N}$ even.

In cyclic symmetry modal analysis, the mode shape in each sector is obtained from the eigenvalue solution. The cyclic symmetry modal solution occurs after the static cyclic symmetry solution, so that the modal analysis yields the mode shapes of the fan blade in the pre-stressed state. The displacement components (x, y, or z) at any node in sector $n$ for harmonic index $k$, in the full fan structure are yielded by Eqn. (6).

If the mode shapes are normalized to the mass matrix in the modal analysis, the normalized displacement components in the full fan blade system are given by,

$$
u_{\text {normalized }}= \begin{cases}\frac{u}{\sqrt{\bar{N}}} & \text { if } k=0 \text { or } k=N / 2 \\ \frac{u}{\sqrt{N / 2}} & \text { otherwise }\end{cases}
$$

In a cyclic symmetry modal analysis, it is necessary to understand the concepts of harmonic indices and nodal diameters. Examining at a corresponding point in each sector, the mode shapes are sinusoidal in the circumferential direction, which indicates nodal lines on the bladed disk system called as nodal diameters (ND). Most mode shapes contain lines of zero out-of-plane displacement which cross the entire disk. This modal behavior is described in more detail in Section III.

In addition to cyclic symmetry constraint equations defined above, surface-to-surface contact pairs were used to define contact behaviors at the blade disk dovetail assembly as illustrated in Figure 3. In the contact between two bodies, the surface of one body was taken as a contact surface and the surface of the other body as a target surface. The "contact-target" pair concept has been widely used in finite element simulations. The contact surface was meshed using contact elements which are a 3D, 8-node, higher order quadrilateral element located on the surfaces of blade dovetail area. The target surface was meshed using target elements which are a 3D, 8-node, higher order quadrilateral to represent 3D target surface for the associated contact elements on the surfaces of disk dovetail socket area. For these surface-to-surface contact elements, the augmented Lagrangian penalty method, ${ }^{[6,9]}$ incorporated with a contact "spring" stiffness called the contact stiffness and target stiffness was used to establish a relationship between the contact-target surfaces for bonded contact in conjunction with cyclic symmetry constraint conditions. Contact problems are highly nonlinear and require significant computer resources. In the present analysis, it was assumed that both contacting bodies are deformable with similar stiffness so flexible-to-flexible contact type was employed. Generally the regions of contact are not known until an actual solution process is evolved with the loads, materials, boundary conditions, and other factors in a largely unpredictable manner.
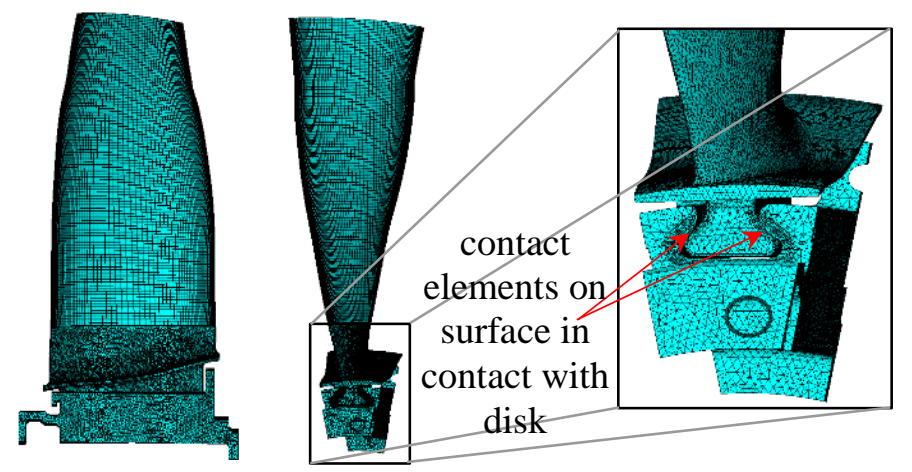

Figure 3: Surface-to-surface contact pairs between disk hub and blade in dovetail attachment

The augmented Lagrangian method used in this study is an iterative series of penalty updates to find the Lagrange multipliers (i.e., contact tractions) and was investigated with the magnitude of the contact stiffness coefficient from a common range of $0.01-1.0$. It was observed from this investigation that the augmented Lagrangian penalty method was insensitive to the magnitude of the contact stiffness coefficient. Hence, this method was employed in the present 
DTF fan forced response analysis, presumably well-suited for a wide range of frictional contact situations governed by a Coulomb law ${ }^{[9]}$ at variable rotor speeds of DTF fan blade. Further boundary conditions are applied at the inner radius of the disk sector to restrain motion in the radial and circumferential directions to prevent the rigid body motions. Also, surface elements manipulated by thickness set to zero were overlaid onto an area face of the blade elements as a modeling technique to apply steady and unsteady aerodynamic pressure loads on the blade surfaces.

In materials used for this analysis, the blade part was made from a titanium alloy and the disk part was made from a steel.

\section{Methods for forced response computation}

A finite element forced response analysis method utilizing the cyclic symmetry modeling approach was employed to provide a comprehensive study of a variety of structural characteristics of a rotating DTF fan blade system. The first task (section A) was to examine the steady stresses including nonlinear rotational effects of spin and stress stiffening due to the centrifugal loading caused by rotational velocity and steady state aerodynamic blade pressure load. The second task (section B) was to perform a prestressed vibration modal analysis, allowing for the classification of every natural mode within the frequency range of interest. This computation also provided information about areas of high stress and how the relative magnitude of these stresses varies with frequency. Finally, section $\mathrm{C}$, a cyclic symmetry finite element analysis model incorporated with Turbo aerodynamic analysis computer program ${ }^{[10]}$ and Ansys structural dynamics computer program was developed to compute the forced response stress distribution in fan blade and as a result the blade structural safety margins for the targeted DTF blade design requirements are presented in section D. While all the equations in the following sections are represented in the cyclic symmetry coordinates, added symbols to the equations are avoided for a brevity in the descriptions.

\section{A. CYCLIC SYMMETRY CENTRIFUGAL STATIC STRESS ANALYSIS MODEL SUBJECTED TO STEADY-STATE AERODYNAMIC PRESSURE AT FAN SPEEDS}

The first task was to develop a cyclic symmetry finite element static analysis model incorporated with Turbo steady-state aerodynamic blade pressure load as a function of the fan blade rotation. To capture the true blade behavior in this rotational condition, geometric nonlinearity was to be accounted for providing the blade deflections potentially to cause significant changes in the blade structural geometry. In such events, while solving the equations of equilibrium by utilizing an iterative process would be employed to obtain the accurate prediction for the largely deformed configuration, excessively high computing time is required for the size of the present problem. Even if a small deflection analysis may not directly account for change in geometry, the effect for large deflection would be possible to be accounted for by an adjustment of the structural stiffness matrix in a small deflection solution approach. This approach allows adjusting the stiffness of a rotating blade to account for dynamic mass effects. Equilibrium of the system and centrifugal forces on the mass using small deflection logic can be expressed as,

$$
[K]\{u\}=\left\{\Omega^{2}\right\}[M]\{r\}
$$

where:

$$
\begin{aligned}
& {[K]=\text { global stiffness matrix }} \\
& \{u\}=\text { displacement of the mass from the rest position } \\
& \{r\}=\text { rest position of the mass } \text { with respect to the axis of rotation } \\
& \Omega=\text { angular velocity of rotation } \\
& {[M]=\text { global mass matrix }=\sum_{e=1}^{e=n} M^{e}} \\
& e=\text { element } 1,2, \ldots, n \\
& {\left[M^{e}\right]=\text { element mass matrix }=\int_{V}[N]^{T}[N] \rho d V} \\
& {[N]=\text { shape function matrix }} \\
& \rho=\text { element density } \\
& n=\text { number of elements }
\end{aligned}
$$

However, to account for deflection effects, Eqn. (8) must be expanded to:

$$
[K]\{u\}=\left\{\Omega^{2}\right\}[M]\{r+u\}
$$

Rearranging terms,

$$
\left([K]-\left\{\Omega^{2}\right\}[M]\right)\{u\}=\left\{\Omega^{2}\right\}[M]\{r\}
$$


Defining:

$$
\begin{aligned}
& {[\bar{K}]=[K]-\left\{\Omega^{2}\right\}[M]} \\
& {[\bar{F}]=\left\{\Omega^{2}\right\}[M]\{r\}}
\end{aligned}
$$

Eqn. (10) becomes simply,

$$
[\bar{K}]\{u\}=[\bar{F}]
$$

$[\bar{K}]$ is the stiffness needed in a small deflection solution to account for large deflection effect, and $[\bar{F}]$ is the same as that derived from small deflection logic. Rotational velocities are combined with the element mass matrices to form a body force load vector. Thus, the large deflection effects are included in a small deflection solution. This decrease in the effective stiffness matrix is called spin-softening. ${ }^{[11]}$ Additionally, with assuming that the latest stress state in the computation may be large enough to change the blade shape under high centrifugal rotation, inclusion of such stiffness effects may need to be examined for the potential changing geometry due to this deformation by large stress which may no longer be neglected. This effect called stress stiffening effect was also included in the computation. This stress stiffening is the stiffening (or weakening) of a structure due to its stress state. This stiffening effect normally needs to be considered for thin structures with bending stiffness very small compared to axial stiffness, such as thin DTF blades which couples the in-plane and out-of-plane displacements. Accordingly, this stress stiffness matrix was added to the stiffness matrix in Eqn. (12) in order to update the total stiffness for accurate predictions. Hence, the equilibrium equation for static analysis is given by,

$$
([\bar{K}]+[\bar{S}])\{u\}=[\bar{F}]
$$

where:

$\{u\}=$ nodal displacement vector

$[\bar{K}]=$ global stiffness including spin softening stiffness due to centrifugal force

$[\bar{S}]=$ stress stiffening (or weakening) due to its stress state $[\bar{S}]=\int_{V}[G]^{T}[\tau][G] d V$ when necessary

$[G]=$ matrix shape function derivatives

$[\tau]=$ matrix of current Cauchy (true) stresses in global coordinate

$[\bar{F}]=$ global load vector due to rotating body force and steady state aerodynamic pressure load

As for the mass term, the mass summary is calculated from accumulating each element contribution which does not reflect the boundary conditions, coupled degrees of freedom, constraint equations, and multipoint constraint in contact elements.

\section{B. PRESTRESSED CYCLIC SYMMETRY VIBRATION MODAL ANALYSIS MODEL SUBJECTED TO CENTRIFUGAL STRESS WITH STEADY-STATE AERODYNAMIC PRESSURE AT FAN SPEEDS}

Given that the cyclic symmetry harmonic forced responses are to be computed in terms of the unsteady state aerodynamic engine order (EO) excitation vibration loads, a perturbed prestressed cyclic symmetry modal analysis model was developed after first performing a cyclic symmetry static analysis for identifying frequencies and mode shapes of blades vibration and critical speed ranges for a given set of nodal diameters (as illustrated in Figure 4). This allows for the classification of every natural mode within the frequency range of interest.

This vibration modal analysis assumed that there is no damping and the structure has no time varying forces applied (i.e., free vibration) to determine the cyclic symmetry natural frequencies and mode shapes. The equation of motion for an undamped system can be expressed in matrix notation,

$$
[M]\{\ddot{u}(t)\}+[\widetilde{K}]\{u(t)\}=\{0\}
$$

where:

$$
[\widetilde{K}]=[\bar{K}]+[\bar{S}]
$$

For a linear system, free vibrations are harmonic of the form,

$$
\{u(t)\}=\{u\}=\{\phi\}_{r} \cos \omega_{r} t
$$


where:

$\{\phi\}_{r}=$ eigenvector representing the mode shape of the $r^{\text {th }}$ natural frequency

$\omega_{r}=r^{\text {th }}$ natural frequency (cycles/second, Hz); $\omega_{r}=\frac{f_{r}}{2 \pi}$ where $f_{r}=$ natural circular frequencies (radians/second)
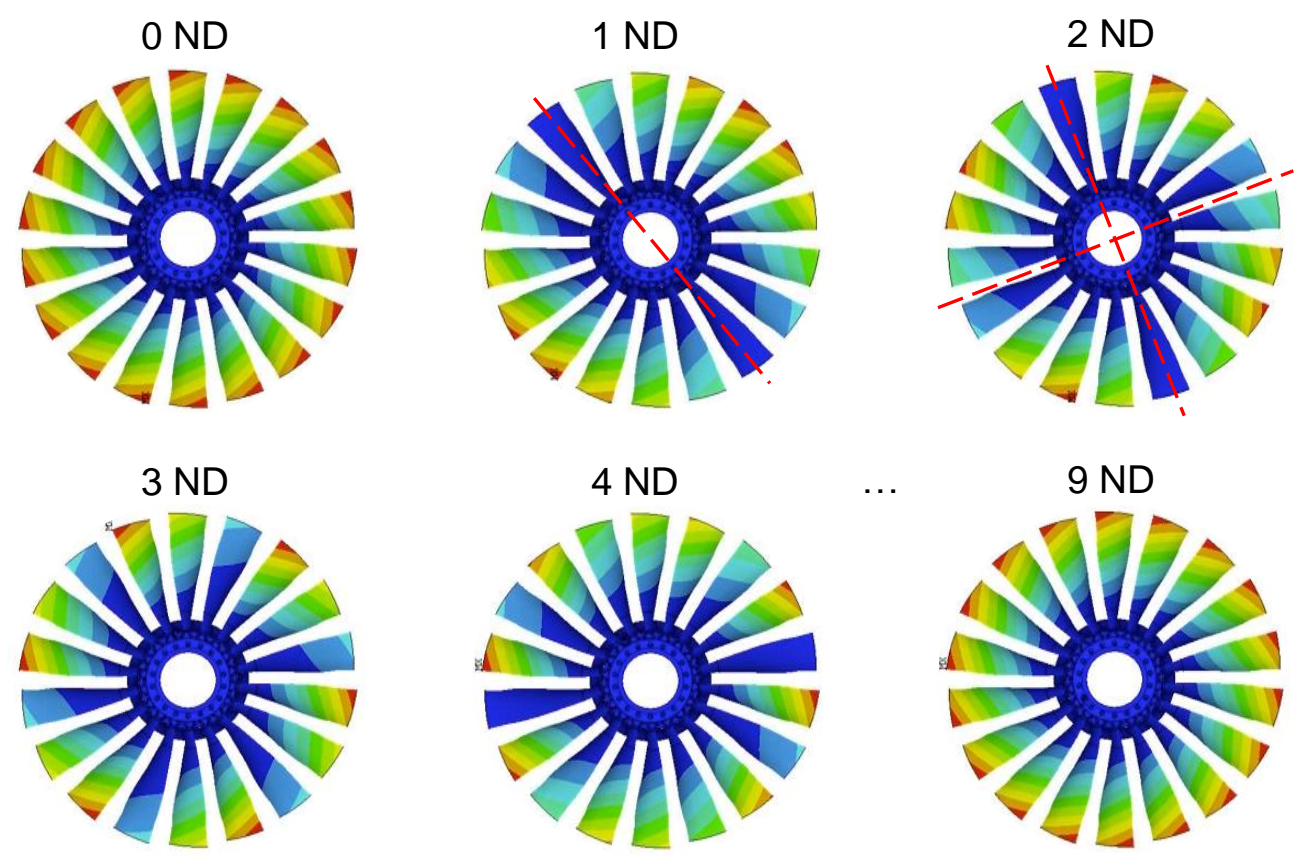

Note: ND = Nodal Diameter; Mode refers to Mode Family; Magnitude of displacement is plotted

\section{Figure 4: Illustration of nodal diameter (ND) patterns from BLI²DTF model shown with higher deformation in red color}

As indicated in Fig. 4, the cyclic symmetry prestressed modal analysis solution provides analytical results for the interaction between the disk vibration and the blade vibration of DTF fan blade. The blade frequencies were solved corresponding to a particular disk mode (or nodal diameter, $N D$ ). Different disk nodal diameter information is to capture the blade frequencies and mode shapes for each nodal diameter.

The cyclic symmetry modal solution uses the same low- and high-edge components defined in the static cyclic symmetry analysis. To calculate the frequencies and mode shapes of a deformed structure, the linear perturbation solution method based on the Newton-Raphson procedure ${ }^{[12]}$ after performing a static analysis is needed for a prestressed modal analysis of cyclic symmetry structures. The linear perturbation modal analysis procedure is designed to solve a problem from this preloaded condition. The linear perturbation modal analysis can be viewed as an iteration solution on top of a prior analysis. During the linear perturbation process, all of the effects from the static analysis are taken into account and are "snapshot solution" so that the perturbed loads can be generated linearly by using the "snapshot solution" matrices and material properties. With a restart procedure from the static analysis, the iterative process is used for a restart solution by allowing modifications of perturbed loads to generate new matrices of perturbation loads, mass density, and damping. The load history vector from the modal analysis is updated for the next iteration solution and subsequently the modal coordinates are updated by solving eigenvalue solution. During this phase of the analysis, the frequency dependent perturbation load vector is calculated and stored in the mode shape file and the element load information files for a subsequent mode-superposition modal-based forced response analysis. This procedure allows for saving significant time in computation and reusing the mode shape information from an earlier modal analysis to improve the accuracy of a mode-superposition harmonic forced response computation. Modal extracting computation process typically requires more time than element loads generation, perturbation residual load vector calculation, and enforced snapshot solution mode calculation.

Hence, the equation of motion for an undamped system can be expressed for the linear perturbation vibration modal analysis to compute the natural frequencies and mode shapes, 


$$
\left(-\omega_{r}^{2}[M]+\left[\widetilde{K}_{l}\right]\right)\{\phi\}_{r}=\{0\}
$$

where:

$\left[\widetilde{K}_{l}\right]=$ stiffness update at the iteration of perturbation load step "l" for prestressed modal analysis where spinsoftening effects and stress stiffening effects included

This equality is satisfied if either $\{\phi\}_{r}=\{0\}$ or if the determinant of $\left(-\omega_{r}^{2}[M]+\left[\widetilde{K}_{l}\right]\right)$ is zero. The first option is the trivial one and, therefore, is not of interest. Thus, the second one gives the solution,

$$
\left|\left[\widetilde{K}_{l}\right]-\omega_{r}^{2}[M]\right|=0
$$

This is an eigenvalue problem which may be solved for up to $n d o f$ values of $\omega_{r}^{2}$ and $n d o f$ eigenvectors $\{\phi\}_{r}$ which satisfy Eqn. (16) where $n d o f$ is the number of degrees-of-freedom (DOFs) of the model.

While several eigenvalue and eigenvector extraction procedures are available for solving Eqn. (17), the present study employed the Block Lanczos method ${ }^{[13]}$ to extract the requested eigenvalues using the sparse direct solving technique that performs forward and backward substitution using the factors. During this solution process, the frequency dependent perturbation load is calculated and stored as nodal forces for a subsequent mode-superposition modal-based harmonic forced response analysis.

Repeat analysis was performed to calculate blade vibration frequencies at different rotational speeds. Also, as will be described in detail in Section IV, an additional objective of this prestressed cyclic symmetry modal analysis was to guide the optimal strain gauge placement on the fan blades by providing the information regarding how the magnitude of modal stresses varies with frequencies so that the optimal locations of the strain gauges are determined on a blade for the effective strain gauge measurement in the experimental wind tunnel test of fan blades.

\section{CYCLIC SYMMETRY HARMONIC FORCED RESPONSE ANALYSIS MODEL SUBJECTED TO A COMPLETE CYCLE OF ENGINE ORDER FORCING VIBRATION BY UNSTEADY-STATE AERODYNAMIC PRESSURE LOAD AT FAN SPEEDS}

Next, a cyclic symmetry harmonic forced response analysis model was developed utilizing the mode superposition method. The results of a cyclic symmetry modal analysis described in Section III.B was retrieved for a harmonic response analysis described in this section. This cyclic symmetry harmonic forced response analysis model allows for a complete solution of DTF fan blade vibration dynamic stresses throughout a complete cycle of engine order forcing vibration.

The finite element semi-discrete equation of motion for rotation including prestressed structural stiffening effects can be expressed in matrix form,

$$
[M]\{\ddot{u}(t)\}+[C]\{\dot{u}(t)\}+\left[\widetilde{K}_{l}\right]\{u(t)\}=\left\{F^{a}(t)\right\}
$$

where:

$$
\begin{aligned}
& {[C]=\text { structural damping matrix }} \\
& \{\ddot{u}(t)\}=\text { nodal acceleration vector } \\
& \{\dot{u}(t)\}=\text { nodal velocity vector } \\
& \{u(t)\}=\text { nodal displacement vector } \\
& \left\{F^{a}(t)\right\}=\text { unsteady aerodynamic EO excitation pressure load } \\
& t=\text { time representing the current equilibrium iteration } \\
& l=\text { load step in perturbation solutions }
\end{aligned}
$$

The cyclic symmetry harmonic forced response analysis solves the time-dependent equations of motion (Eqn. (18)) for DTF fan blade structures undergoing harmonic forced vibration. This analysis assumed that the entire DTF blade structure has frequency-dependent stiffness, damping, and mass effects and all loads and displacements vary sinusoidally at the same known frequency (although not necessarily in phase). Mathematically, Eqn. (18) represents a system of linear differential equations of second order. Two procedures can be considered for the solution of Eqn. (18): direct integration method and mode superposition method. Given that a traveling wave EO excitation occurs due to circumferential disturbances in the BLI flow field from upstream of the fan and a mode superposition method shows the numerical effectiveness over a direct integration method, engine order loading (circumferentially traveling wave excitation) is modeled with a mode-superposition analysis method. ${ }^{[6,14]}$ Engine order excitation is periodic 
forcing arising from circumferential disturbance in a set of blades on the bladed rotor. The mode-superposition method sums factored mode shapes using the natural frequencies and mode shapes obtained from a linear perturbed modal analysis to characterize the dynamic response of a harmonically excited structure. In addition, since a mode superposition method may need to consider only a few modes, the mode superposition method can be much effective than a direct integration which would be prohibitively expensive in computations if the order of the matrices is large. ${ }^{[14]}$

Eqn. (18) was solved by the mode-superposition method to the interest of specific EO exciting unsteady aerodynamic pressure load on the blades. $\left\{F_{a}\right\}$ is the time-varying load vector given by,

$$
\left\{F_{a}\right\}=\left\{F^{n d}\right\}+s f \cdot\left\{F^{s}\right\}
$$

where:

$\left\{F^{n d}\right\}=$ time varying unsteady aerodynamic nodal forces

$s f=$ load vector scale factor

$\left\{F^{s}\right\}=$ load vector read in from the linear perturbation modal analysis in Section III.B

Note that all loads from the linear perturbation modal analysis are scaled, including forces and pressures. All loads from the modal solution load vector during a given load step in the linear perturbation modal analysis is applied as engine order loads. Damping in Eqn. (18) should be specified; otherwise, the response will be infinite at the resonant frequencies. Additional information on the transformation of Eqn. (18) from cyclic symmetry coordinates to physical coordinates and Fourier transformation in cyclic symmetry forced response analysis are described in reference 6.

Hence, similarly given in reference 14, a general treatment of dynamic systems can be formulated by Lagrange's equation from the scalar quantities of kinetic energy, potential energy, and work where the equations of motion of a system can be formulated in a number of independent coordinate systems. Such independent coordinates are called generalized coordinates and can be denoted by the character $q_{r}$ in the present study. Eqn. (18) can be transferred into modal space and hence, a set of modal coordinates $q_{r}$ was defined such that,

$$
\{u\}=\sum_{r=1}^{n \bmod }\left\{\phi_{r}\right\} q_{r}
$$

where:

$\left\{\phi_{r}\right\}=\mathrm{r}^{\text {th }}$ mode shape

nmod $=$ number of modes to be used from the preceding modal analysis in Section III.B

Substituting Eq. (20) into Eqn. (18)

$$
[M] \sum_{r=1}^{n \bmod }\left\{\phi_{r}\right\} \ddot{q}_{r}+[C] \sum_{r=1}^{n \bmod }\left\{\phi_{r}\right\} \dot{q}_{r}+\left[\widetilde{K}_{l}\right] \sum_{r=1}^{n \bmod }\left\{\phi_{r}\right\} q_{r}=\left\{F^{a}(t)\right\}
$$

After few steps of the matrix transformation and substitution with applying its orthogonality and normality conditions, and only the $r=j$ terms remain, Eqn. (21) can be expressed in the modal coordinates as,

$$
\ddot{q}_{J}+2 \omega_{j} \xi_{j} \dot{q}_{J}+\omega_{j}^{2} q_{j}=f_{j}
$$

where:

$q_{j}=$ displacement in modal coordinates in terms of harmonic indices

$f_{j}=$ force in modal coordinates in terms of harmonic indices

$\xi_{j}=$ fraction of critical damping for mode $j$

$\omega_{j}=$ natural frequency of mode $j$

Since $j$ represents any mode, Eqn. (22) represents nmod uncoupled equations in the nmod unknowns $q_{j}$. The advantage of this uncoupled system with the mode-superposition method is that all the computationally expensive matrix algebra has already been done in the eigenvalue solver, so extensive time-dependent equations of motion (Eqn. (18)) can be analyzed inexpensively in modal coordinates along with Eqn. (20). The modal coordinates $q_{j}$ are converted back into the system response displacements $\{u\}$ to the applied loading using Eqn. (20). In other words, the individual modal responses $q_{j}$ are superimposed to obtain the actual system response (i.e., mode-superposition). This set of uncoupled cyclic sector equations is solved while enforcing the compatibility boundary conditions between the sectors. In a harmonic forced vibration, the force term can be expressed as,

$$
f_{j}=f_{j c} e^{i \Omega t}
$$


where:

$f_{j c}=$ complex harmonic force amplitude

$\Omega=$ rotor engine order (EO) excitation frequency $(\mathrm{Hz})$

Similarly,

$$
q_{j}=q_{j c} e^{i \Omega t}
$$

where:

$q_{j c}=$ complex amplitude of the modal coordinate for mode $j$

Solving for $q_{j c}$ in Eqn. (22),

$$
q_{j c}=\frac{f_{j c}}{\left(-\Omega^{2}+i 2 \omega_{j} \Omega \xi_{j}+\omega_{j}^{2}\right)}
$$

Finally, the complex forced harmonic response displacements (similarly for stresses) are obtained from Eqn. (20) as,

$$
\{u\}=\left\{u_{c}\right\}=\sum_{j=1}^{n m o d}\left\{\phi_{j}\right\} q_{j c}
$$

where:

$$
\left\{u_{c}\right\}=\text { complex displacement vector }
$$

The maximum response will occur when this excitation frequency crosses a natural frequency of the specified nodal diameter.

\section{METHOD FOR SAFETY MARGIN PREDICTIONS USING CALCULATED FORCED RESPONSE STRESSES}

Lastly, a procedure to calculate the safety margin on fatigue to failure was developed by using a Goodman diagram $^{[15]}$ approach to predict the safety margins of the DTF fan blades, as illustrated in Figure 5.

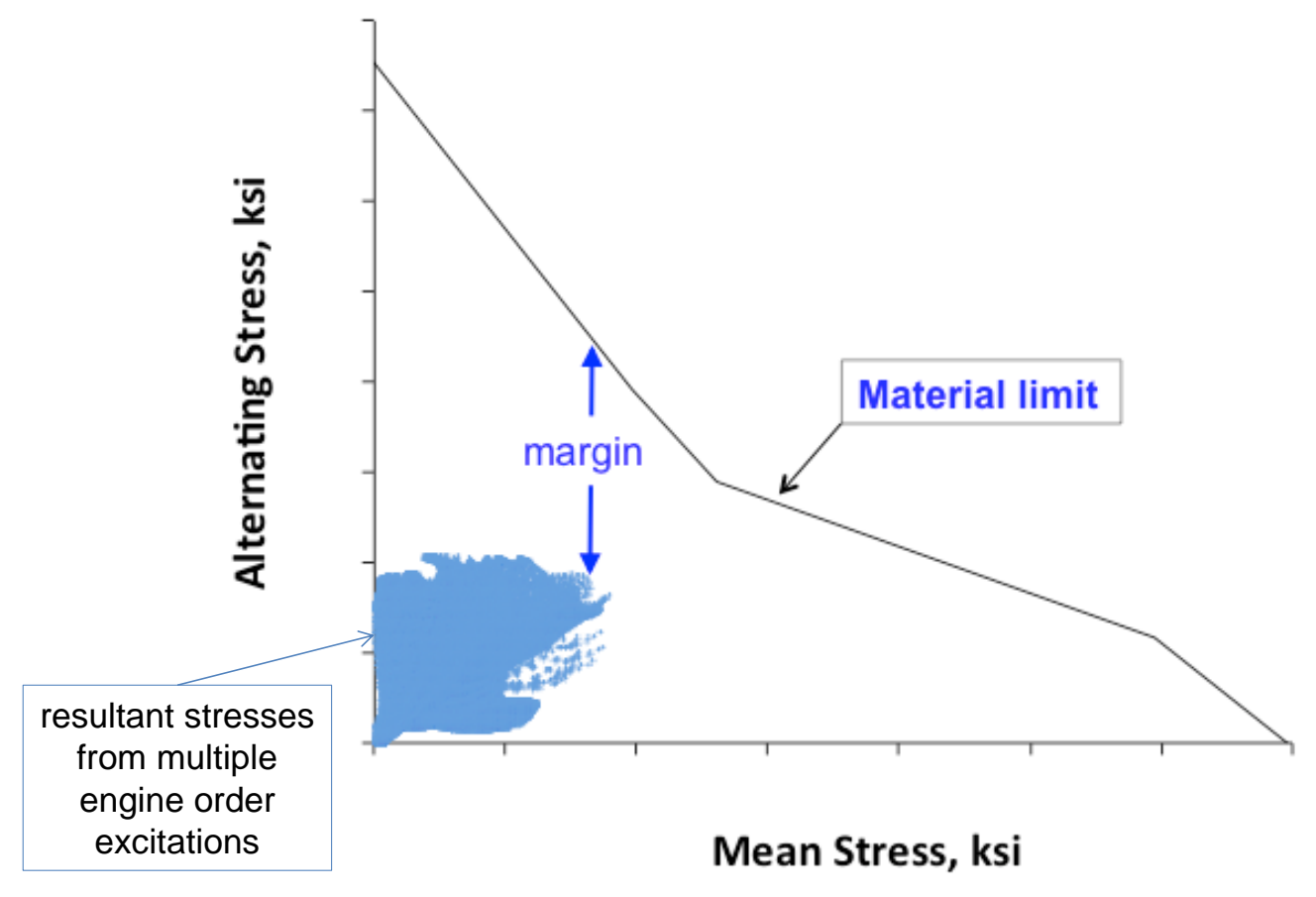

Figure 5: Illustration of Goodman diagram employed in this study 


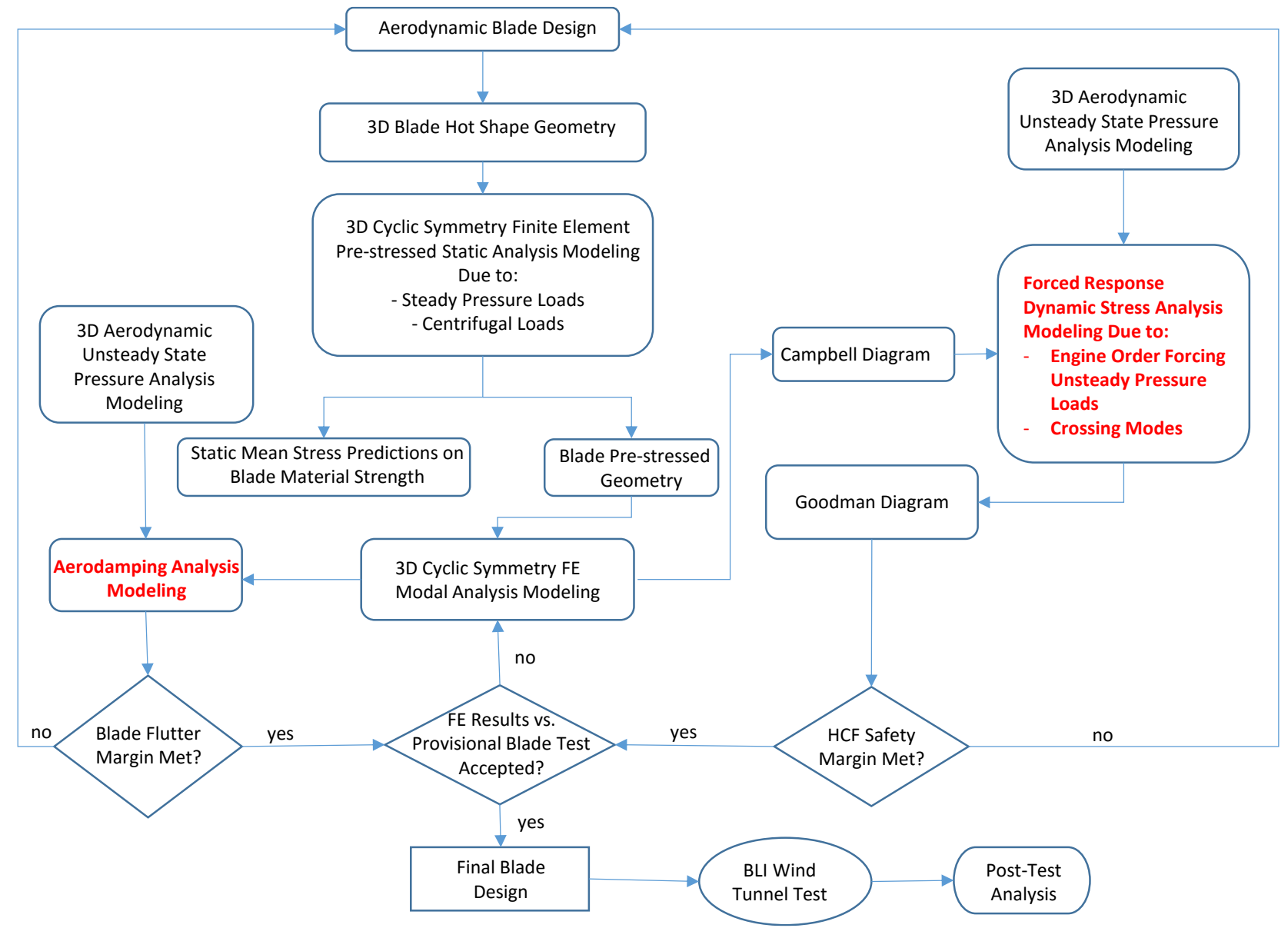

Figure 6: Flow chart showing evolving development of present aeromechanics design analysis models

The general trend given by the Goodman relation is described as decreasing fatigue life with increasing mean stress for a given level of alternating stress (dynamic stress). The relation can be plotted to determine the safe cyclic loading of a part. If a coordinate value indicated by the mean stress and the alternating stress lies under the given material strength curve then the part will survive. If a coordinate value is above the curve then the part will fail for the given stress. While the conventional Goodman relationship may be expressed by the equations in reference 15, an alternative Goodman diagram was utilized from the actual material testing provided by the material supplier as illustrated in Figure 5 for the present analysis. A flow chart of the framework components of the present computational procedures evolving development of present aeromechanics design analysis models is briefly given in Figure 6 .

\section{Determination of blade strain gauge (SG) locations}

Strain gauges for monitoring blade vibrations were placed where high dynamic strain was expected for the structural modes and engine orders of interest (based on aeromechanics analyses), and similarly for monitoring flutter. The optimal strain gauge locations were determined from the modal strain plots as illustrated in Figure 7. SG locations with very high gradients were avoided. Experimental test software was also used to optimize placement of a given number of gauges to capture modes of interest. 

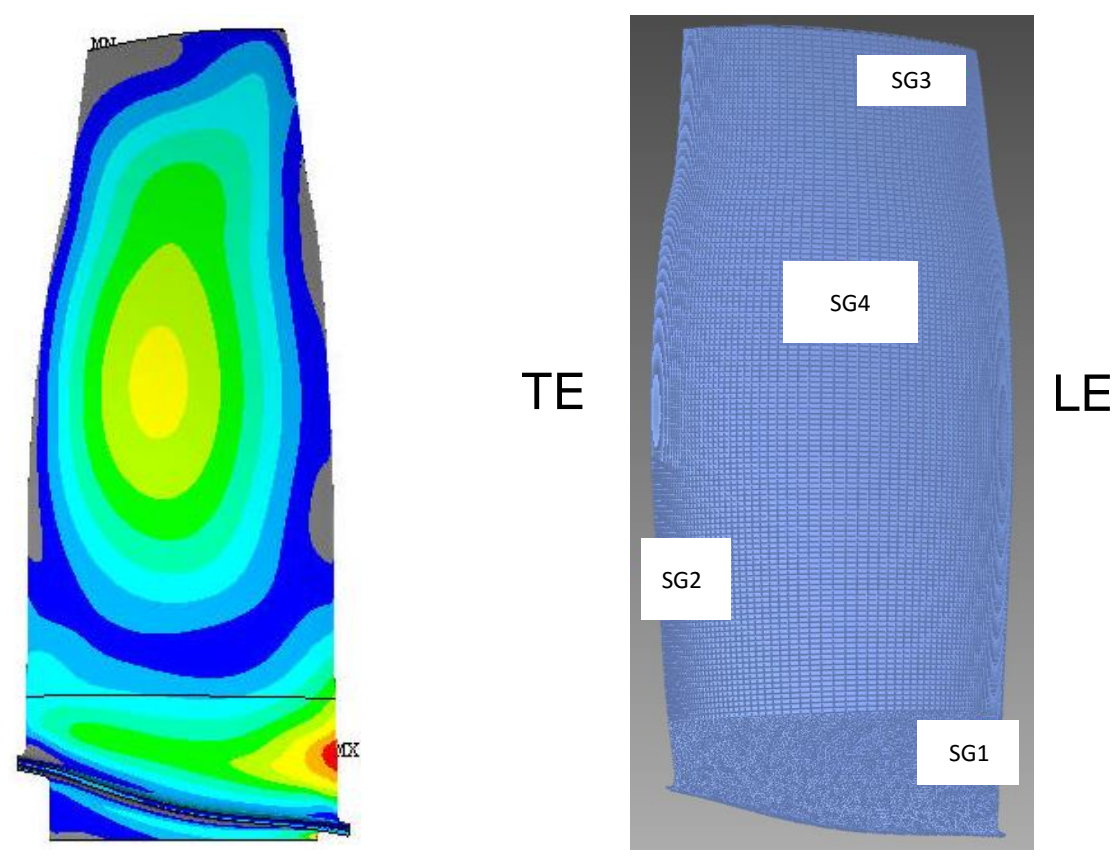

Figure 7: Illustration for determination of strain gauge locations on $B L I^{2} D T F$ finite element mesh

\section{Result and comparison}

\section{A. STATIC MEAN STRESS ANALYSIS RESULT SUBJECTED TO CENTRIFUGAL LOAD AND} STEADY-STATE AERODYNAMIC PRESSURE LOAD AT FAN SPEEDS

Figure 8 shows the steady-state aerodynamic pressure distribution on each side of the blade calculated using Turbo aerodynamic analysis computer program. This pressure load was applied together with centrifugal forces at the fan design speed to compute the static mean stresses.
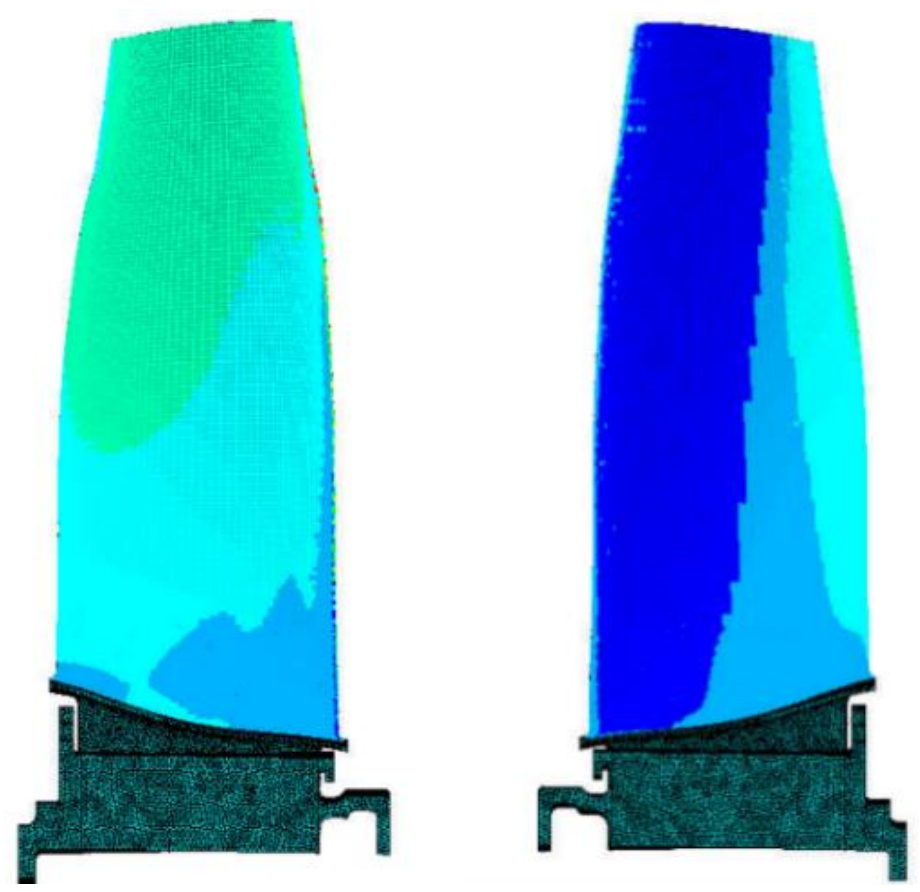

Figure 8: Steady-state aerodynamic pressure distribution contours on blade pressure surface (left) and suction surface (right) computed using Turbo aerodynamic analysis computer program 
Different regions of the blade including dovetail hub area of the airfoil undergo high centrifugal stresses when the blade spins along with the steady-state aerodynamic load. As the state of stress is multiaxial, von Mises stress was considered to identify the site of higher mean stress in the blade. Figures 9 and 10 display the results of steady state displacements and stresses on the fan surfaces. These contour plots provide information on the mean stress amounts along with the vibratory stresses obtained from a process of the forced response computations. This information is used to calculate the safety margins of the DTF fan blade using a Goodman diagram shown in Figure 5 in Section III.D. It was also observed in this analysis that maximum deflection occurs at the blade leading edge tip as shown in Fig. 9.

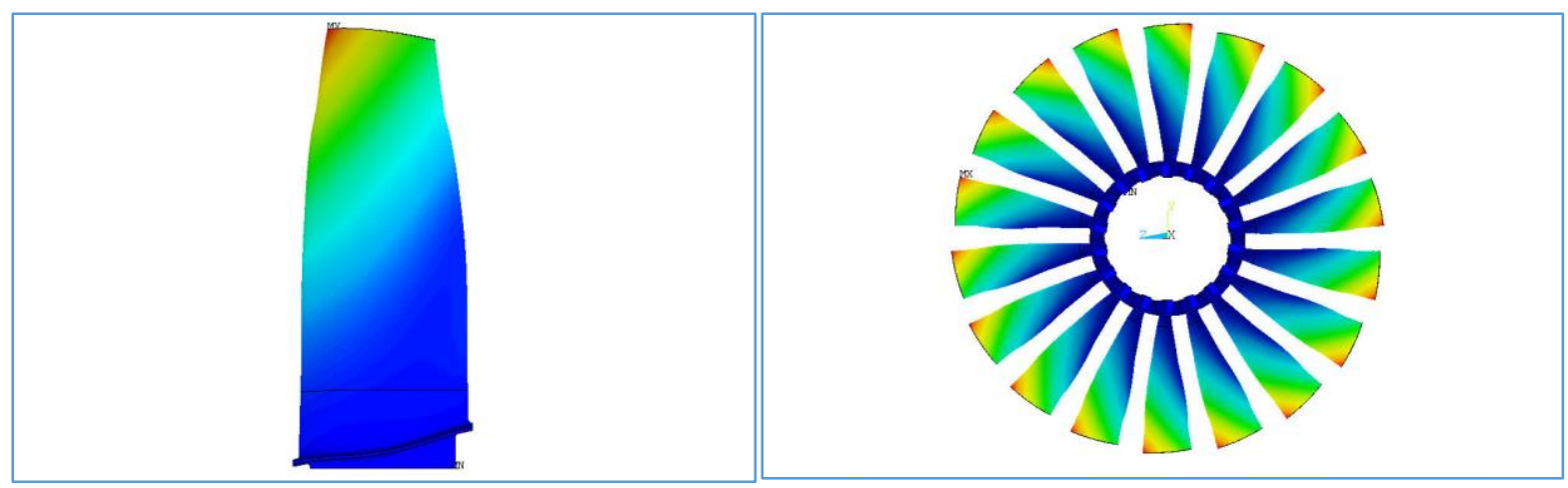

Figure 9: Vector sum of displacements yielded by rotation and steady-state aerodynamic pressure load on single blade (left) and on a full blades rotor (right)
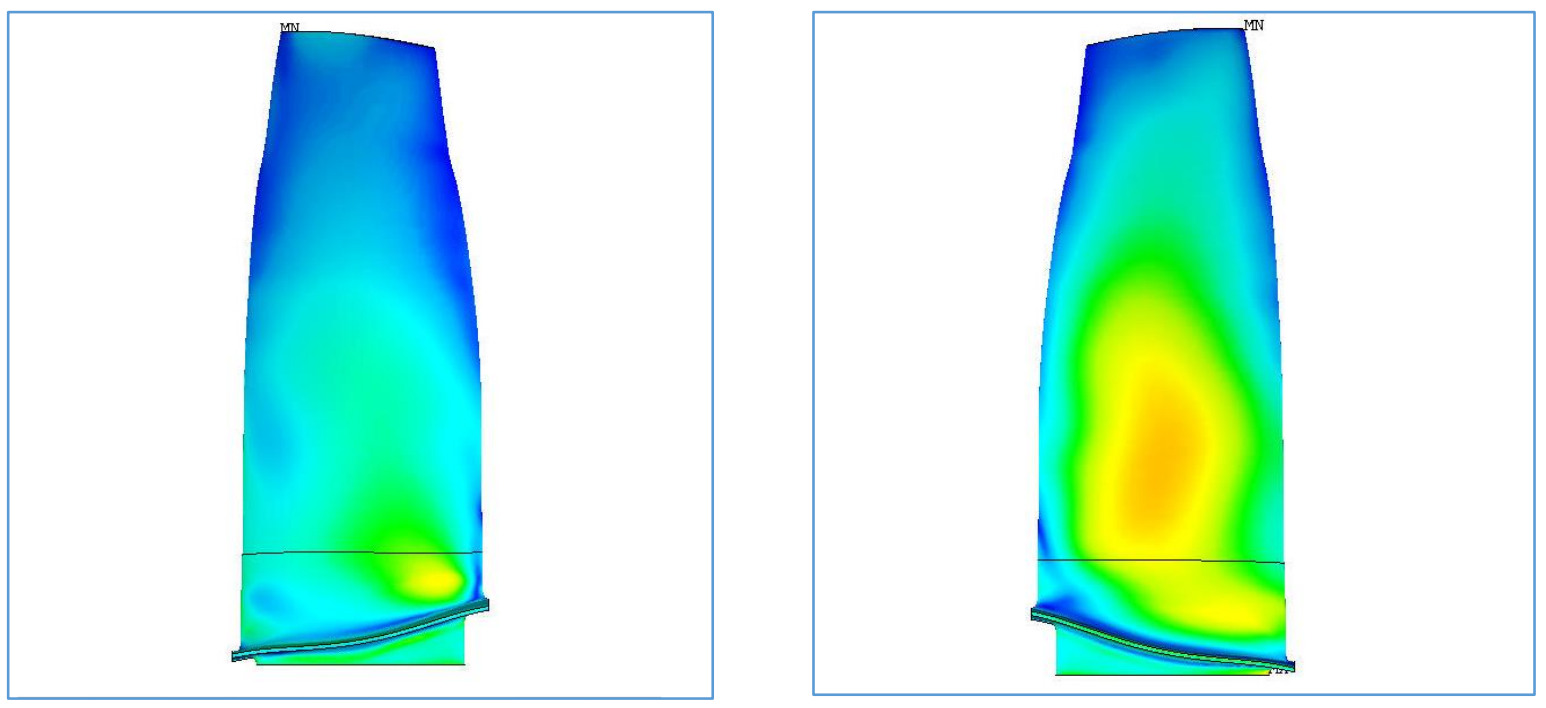

Figure 10: Static mean stresses on suction surface (left) and on pressure surface (right)

\section{B. PRESTRESSED CYCLIC SYMMETRY VIBRATION MODAL ANALYSIS RESULT SUBJECTED TO CENTRIFUGAL STRESS WITH STEADY-STATE AERODYNAMIC LOAD AT FAN SPEEDS}

As described in section III.B, the cyclic symmetry modal analysis was carried out at different speeds for different modes to study the blade vibration harmonic modal characteristics for individual nodal diametric behavior. Figures 11-13 show the mode shapes and frequencies for different nodal diameter (ND) patterns of the DTF fan blades. Note that Figures 11-13 are given only to illustrate the pattern changes of the mode shapes for a different mode family. 


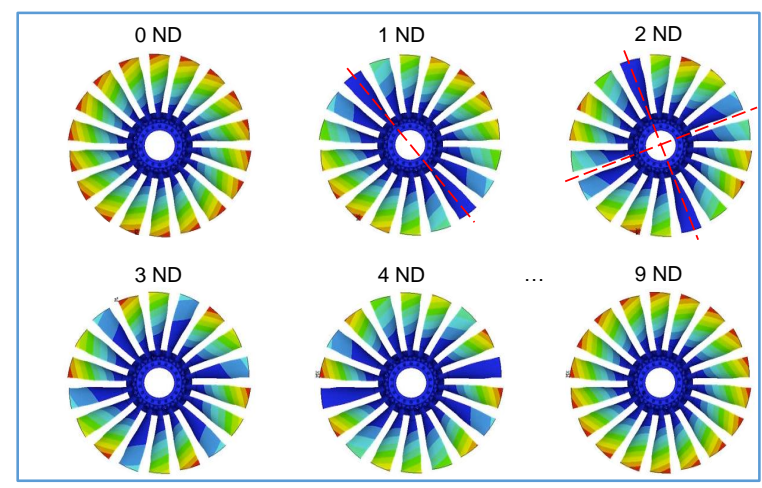

Figure 11: Fan blade mode shape (mode 1)

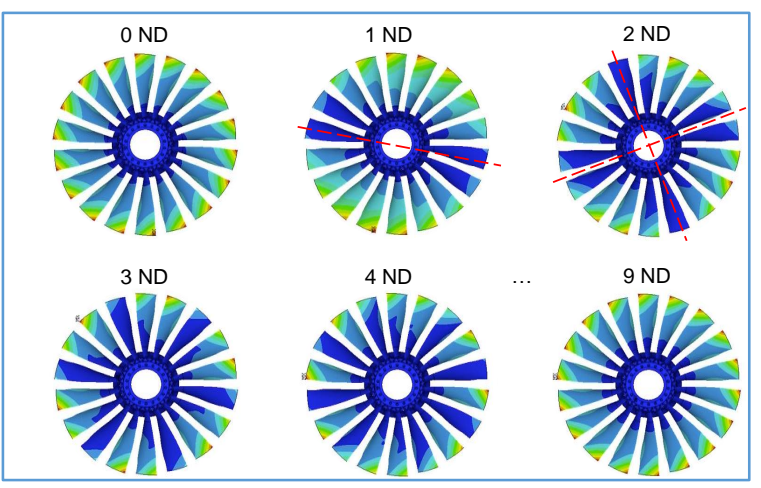

Figure 12: Fan blade mode shape (mode 2)

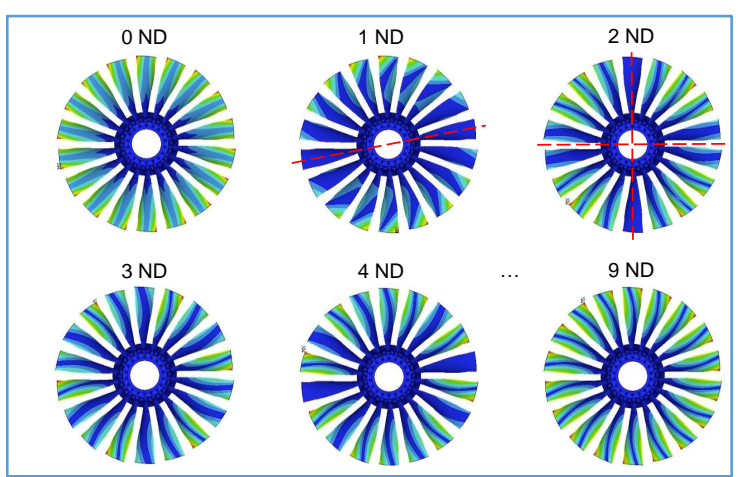

Figure 13: Fan blade mode shape (mode 3)

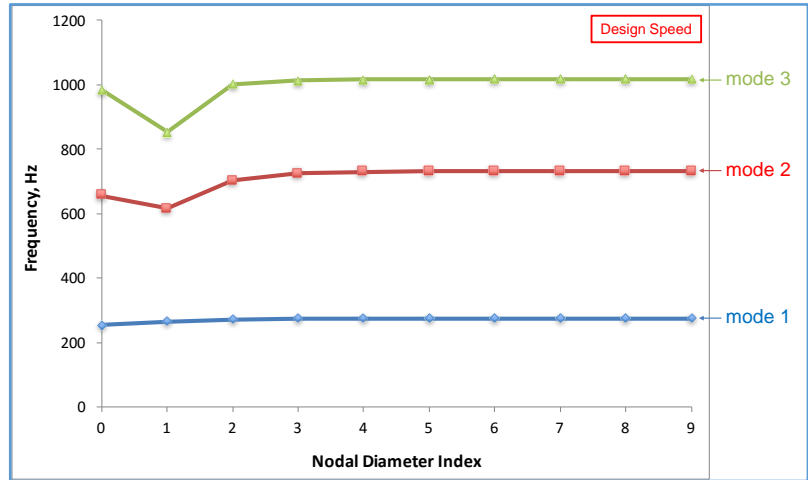

Figure 14: Natural frequency map of $\mathrm{BLI}^{2} \mathrm{DTF}$ for first three modes in terms of nodal diameters

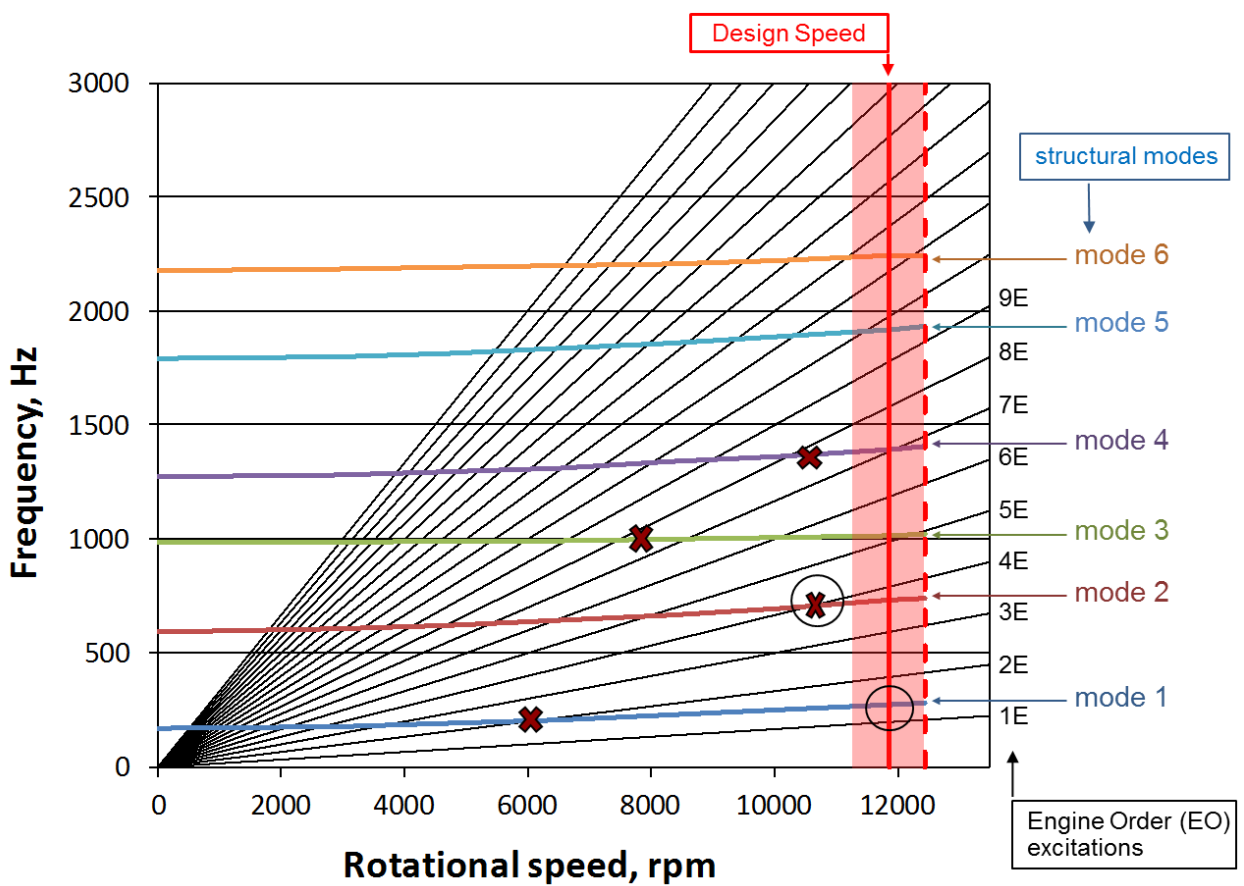

Figure 15: Campbell diagram of BLI'DTF: analysis (lines in colors) vs. blade spin rig test ("x" in symbol) 
Figure 14 shows the free vibration natural frequencies of the fan in terms of the number of nodal diameters in the corresponding mode shapes, which is considered as the frequency map to assist whether the families of modes with frequencies interact with each other (so called frequency coupled veering behaviors) or isolated families of modes. This information provides the vibration characteristics of fan blades. As indicated in this frequency map, the modes of the current DTF fan blade system display fairly isolated between the modes, so that only the modes with natural frequencies near that of the mode of interest would contribute significantly to the forced responses.

Next, frequencies were plotted on a Campbell diagram as shown in Fig. 15 to determine operational engine speeds which may be high-risk in terms of structural resonant vibrations. This Campbell diagram contains the blade rotor speed, the various engine orders (EO or E) of excitation, and the blade rotor natural frequencies with respect to its rotational speed and nodal diameter, obtained through a computational method in Section III.B. A Campbell diagram plot is a mathematically constructed diagram used to check for coincidence of vibration sources with the DTF fan blade natural frequencies. Change in blade natural frequencies with respect to engine speeds are plotted in the diagram. The horizontal lines represent the natural frequencies of the DTF fan blade system in terms of operational speeds. The natural frequencies of each mode are slightly elevated as the operation speed increases, which is attributed primarily due to the radial stress effect in tension. The straight inclined lines represent the EO forcing frequencies as a function of blade rotor speed. Critical speed is defined as the operating speed where the EO excitation frequency equals the blade natural frequency. At critical speed, the fan blade vibration amplitudes may increase extremely high. These critical speeds can be identified by constructing a Campbell diagram. Because the critical speeds are determined graphically, their accuracy depends upon the quality of the Campbell diagram. This diagram promptly leads to determine the risk of resonant conditions at various operating engine speed ranges. Note that as mentioned earlier the results of the present cyclic symmetry modal analysis modeling were compared against experimental spin rig test data in Figure 15. As indicated in this figure, the natural frequencies computed by the prestressed cyclic symmetry modal analyses carried out at different speeds ranging from 0 to DTF fan blade design speed show good agreements with those measured by the blade spin rig experimental test which are shown in symbol " $x$ " on the diagram. This Campbell diagram also shows that a resonant condition so called "crossing" is not predicted at a DTF fan blade "design speed" condition. However, in computational analysis and experimental test it was considered that Mode 2 may be resonant with a 4EO excitation at "off-design speed" condition. Hence, this offdesign speed condition was also investigated for the stress amplitudes when the resonance occurs for a correlation.

\section{CYCLIC SYMMETRY HARMONIC FORCED RESPONSE ANALYSIS RESULT SUBJECTED TO A COMPLETE CYCLE OF ENGINE ORDER FORCING VIBRATION BY UNSTEADY-STATE AERODYNAMIC PRESSURE LOAD AT FAN SPEEDS}

Due to inlet distortions and circumferential variations in the DTF fan blade system, each vibration mode experiences non-axisymmetric periodic excitation forces with a frequency being multiple of the rotational speed. In the present analysis, particularly dynamic stress amplitudes of a condition of $1 \mathrm{EO}$ excitation as an off-resonance case and a potential resonant crossing case of 4EO condition on Mode 2 were great concerns in both blade design process and a validation of analytical modeling. Hence, the forced response analysis was carried out to investigate the 1EO condition at a DTF fan design speed which is depicted by a modal strain contour plot of Mode 1 in Figure 18, and the $4 \mathrm{EO}$ condition at a resonant crossing speed (4EO, Mode 2) in Figure 19. The analysis results are described in this section and compared with those measured from the wind tunnel experimental test.

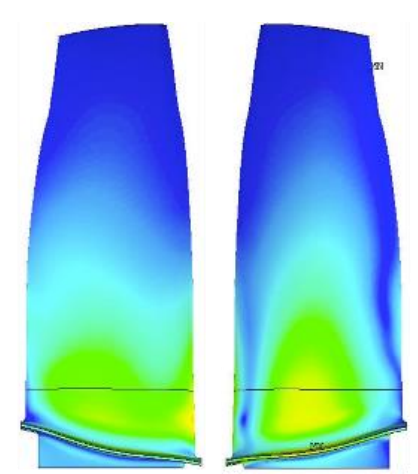

Figure 18: Modal strain for Mode 1 ND1 (1EO)

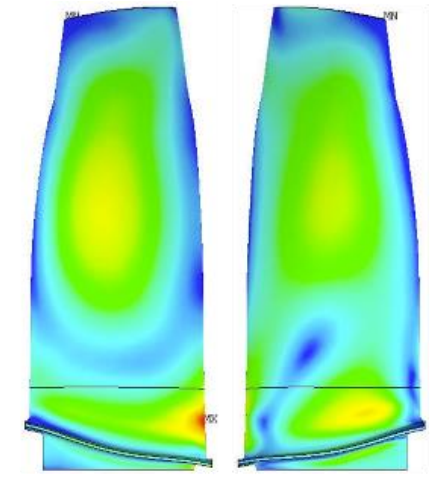

Figure 19: Modal strain for Mode 2 ND4 (4EO) 


\section{C.1) Forced response at design speed at 1EO unsteady aerodynamic excitation pressure (off-resonance}

response)

A cyclic symmetry harmonic forced response analysis was performed for the $1 \mathrm{EO}$ unsteady pressure excitation at the DTF fan blade design speed.

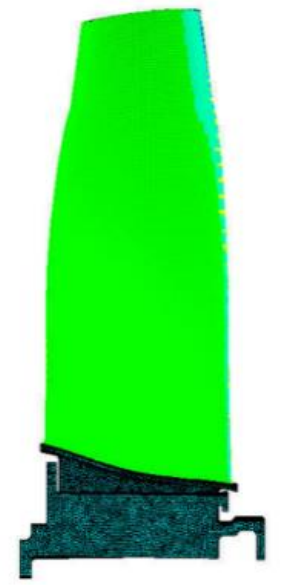

(a) Pressure side-Real

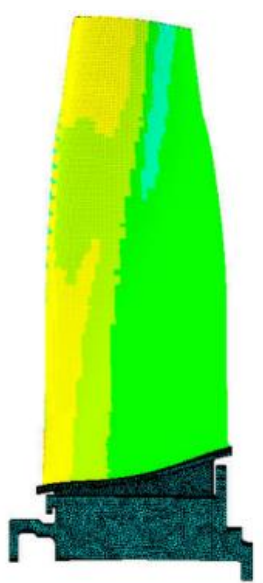

(b) Suction side-Real

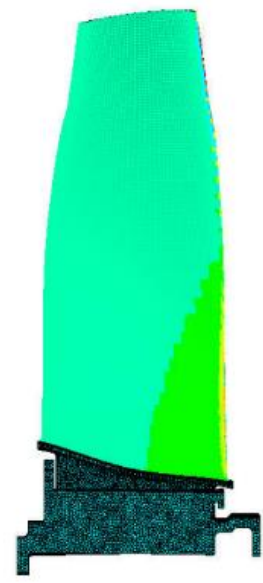

(c) Pressure side-Imaginary

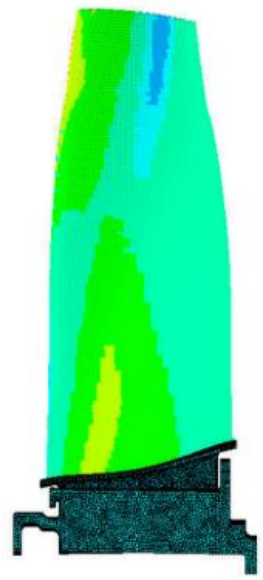

(d) Suction side-Imaginary

Figure 20: Representative unsteady real and imaginary aerodynamic loads on blade pressure surface (a, c) and suction surface $(b, d)$ for $1 \mathrm{EO}$ excitation condition

The alternating stresses from engine order excitations $1 \mathrm{EO}$ can be considered as off-resonance since the excitation frequency is not close to a mode natural frequency (i.e. the frequency margin which is the ratio of the difference between blade natural frequency and the nearest excitation frequency to the blade natural frequency is large) as indicated in the Campbell diagram in Fig. 15. Thus, for this forcing excitation case, damping will not significantly affect the calculated stresses. Even so minimal damping of 0.03 percent was used to prevent the ill-condition when computationally solving the harmonic forced response equations. The unsteady fan blade surface pressure loads represented by the contours of 1EO excitation condition are shown in Figure 20 and applied in the response analysis.

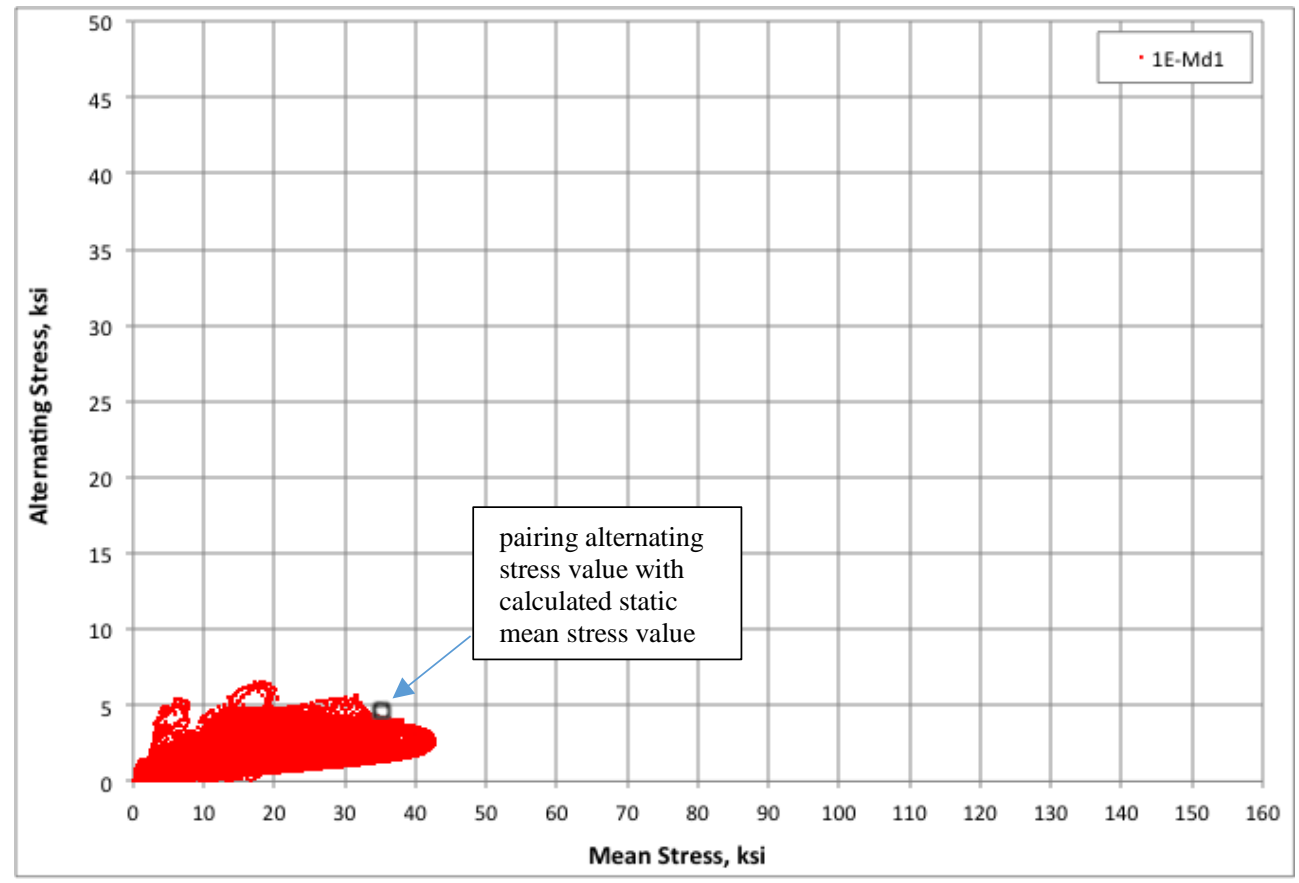

Figure 21: Responses to an excitation at 1EO frequency illustrated using a case of Mode 1 contribution 
Since the state of stress is considered as multiaxial in the DTF fan blade operating condition, von Mises stress was examined in the forced response analysis as illustrated in Figure 21 which displays stress values at every node of the finite element nodal locations on the blade model. Figure 21 is depicted as an illustration purpose of the present analysis approach using a case of $1 \mathrm{EO}$, Mode 1 solution prior to including contributions by a number of modes excited at $1 \mathrm{EO}$ frequency in the present mode superposition computations.

As mentioned in Section V.B, the modes of the present DTF fan blade system are fairly isolated between the modes, so that only the modes with natural frequencies near that of the mode of interest could contribute significantly to the response. The effects of the number of modes in mode superposition computations were examined for this offresonance condition.

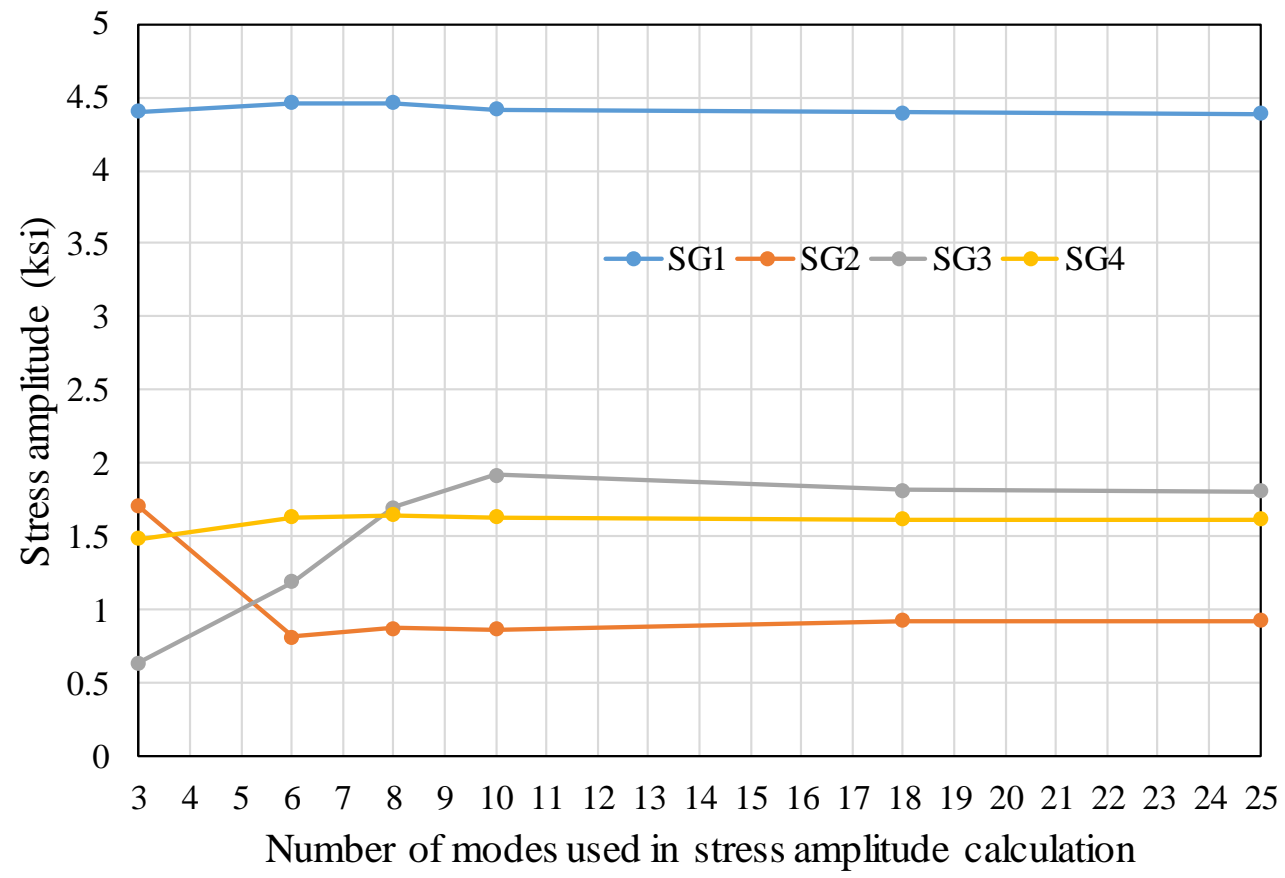

Figure 22: Influence of the number of modes at SG locations at 1EO unsteady aerodynamic excitation

Many modes are often necessary to extract an accurate mode superposition solution. The effectiveness of mode superposition method depends on the number of modes that must be included in the analysis. Thus, if not enough modes are considered, Eqn. (18) may not be solved accurately enough. However, in general only a relatively small set of the fundamental modes would be sufficient to accurately calculate the forced responses. The ultimate aim in the present analysis is to obtain a good approximation to the actual exact response of the DTF fan blades. Figure 22 shows the result of the individual modal responses superimposed to obtain the blade vibratory responses at design speed due to BLI distortion at four strain gauge locations in the direction of the gauge. This result suggests that a numerically converged forced response can be obtained using a moderate number of modes superimposed in the mode superposition forced response analysis. Hence, the present analysis has superimposed 25 modal responses, resulting in the forced response dynamic stress amplitudes to be compared with those measured from the wind tunnel experimental test.

Figure 23 shows a comparison of unsteady stress amplitudes between analysis and wind tunnel test ${ }^{[16]}$ for a condition of $1 \mathrm{EO}$ unsteady aerodynamic loading excitation. The forced response analysis result and measured data from the wind tunnel experimental test at SG locations are presented in this figure. Both the analysis and experimental test results indicated good match while the experimental measurement varies to some degree. Note that averaged values of the measured data are also displayed along with the range of the values of individual SG. 


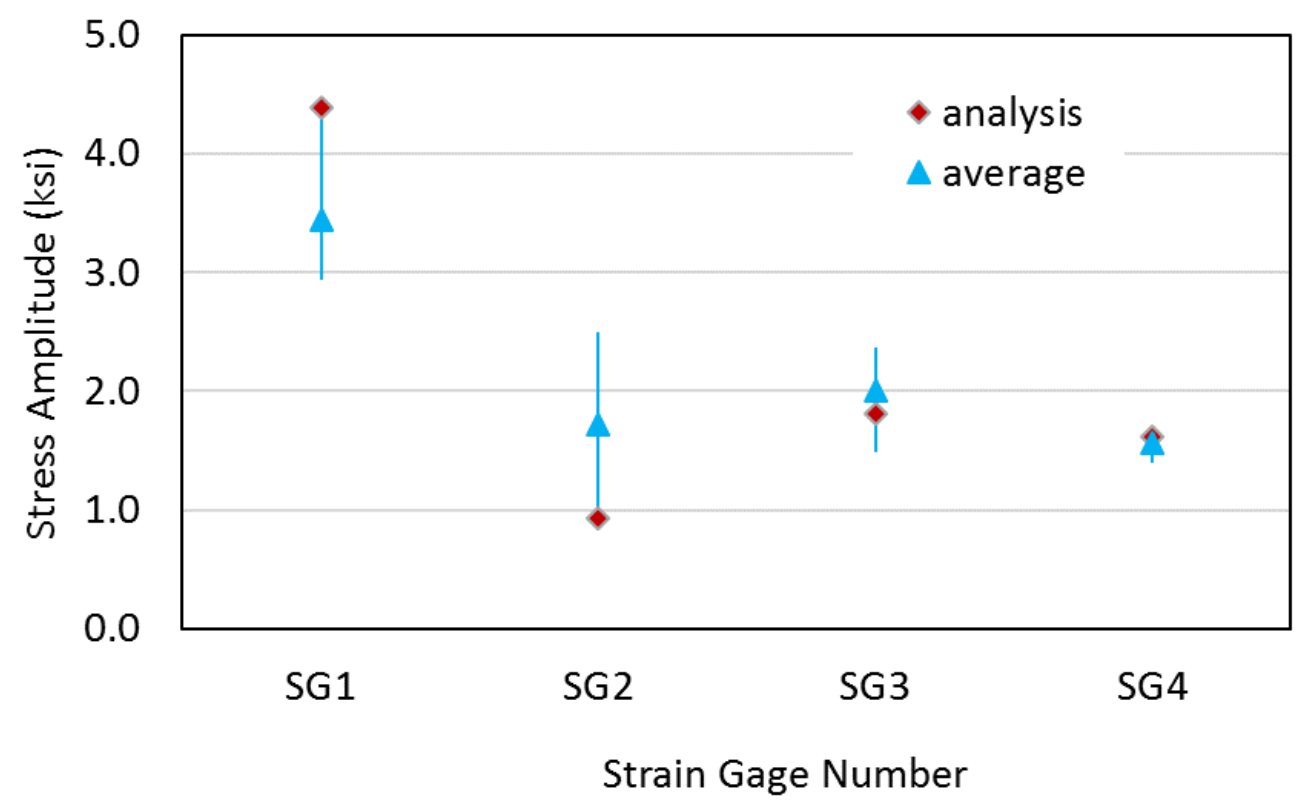

Figure 23: 1EO forced response vibratory stress at design speed: measurement vs. analysis

\section{C.2) Forced response subject to a resonant crossing speed for 4EO, Mode2 (resonance response)}

As indicated in Figure 15, for variable fan speeds, vibratory stresses need to be calculated for all possible resonant conditions over the operating speed range of the DTF fan blades. Nevertheless, as a verification purpose, a 4EO Mode 2 crossing condition was investigated for the stress amplitudes when the resonance occurs.

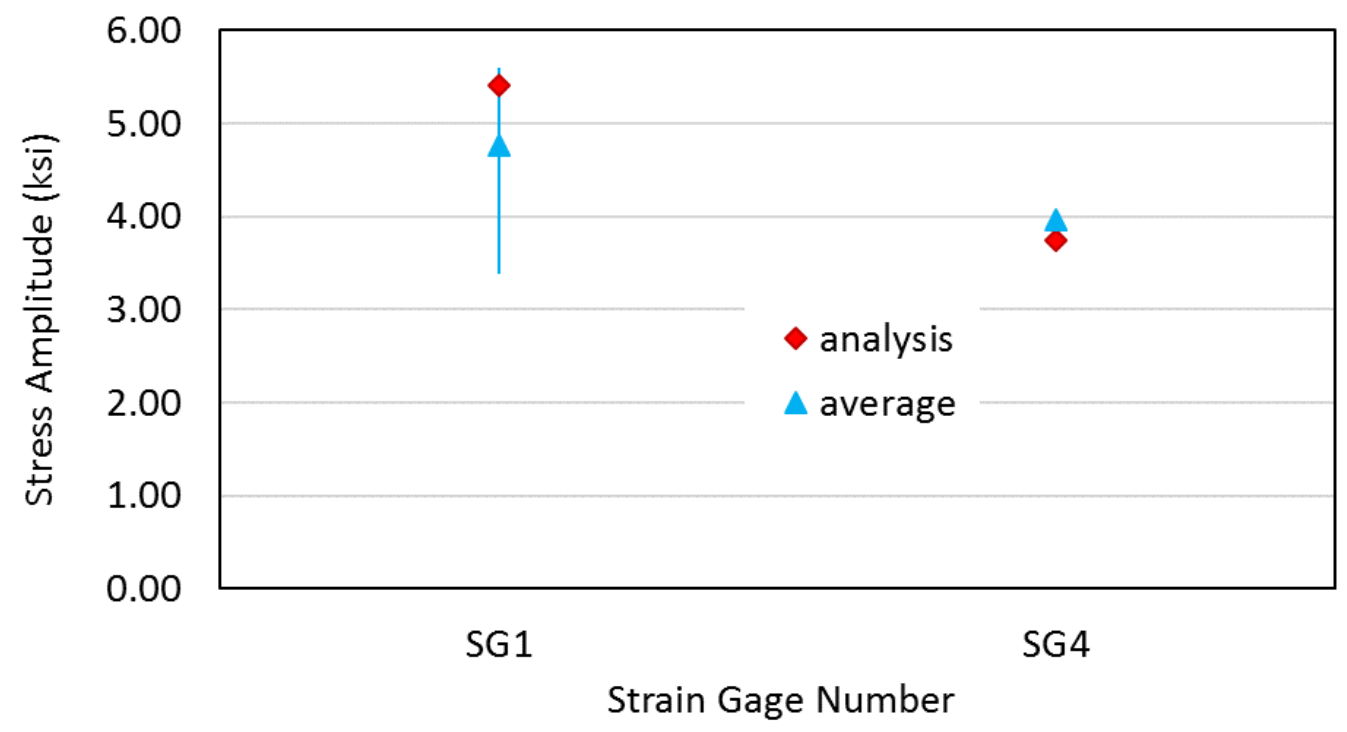

Figure 24: Resonant stress at 4EO, Mode 2 crossing: measurement vs. analysis 
The damping ratio at this resonant speed was available from the experimental test as 1.0 percent ${ }^{[17]}$ and its unsteady aerodynamic analysis result and the wind tunnel measurement data were also available for this specific condition. Unsteady aerodynamic loading was from Turbo analysis at 85\% speed with BLI distortion upstream of the fan. Hence, forced response analysis for this condition was performed and the analysis results were compared with the wind tunnel measurement. It is also considered that responses will be higher with rakes. This analysis helps to understand the frequencies and amplitudes of vibration when this resonance occurs.

Figure 24 shows a comparison between analysis and wind tunnel test. The forced response analysis result and measured data from the wind tunnel experimental test at SG are presented in this figure. Both the analysis and experimental test results indicated good match while the experimental measurement also varies to some degree as indicated for an off-resonance response in Figure 22. The forced response analysis for this potential resonance condition indicated that this resonance condition was considered to be acceptable in terms of a comparison to an offresonance stress amplitude at design speed. Note that measurement data of SG2 and SG3 were unavailable when this correlation was made so a comparison of SG2 and SG3 is not presented in this figure.

\section{FATIGUE MATERIAL MARGIN PREDICTIONS USING FORCED RESPONSE RESULTS}

Forced response analyses determined the dynamic stresses in DTF blade due to continual operation in a distorted flow resulting from BLI. The present cyclic symmetry forced response analysis parallel with the experimental test measurement found a good match in results as indicated in Sections V.C.

Next, these stress values from static and dynamic analysis models were used for fatigue life assessment. It was considered that the present DTF blade made from ductile titanium material under combined bending, torsion, or multiaxial type of the fatigue follows a distortion-energy yield criterion (von Mises). In the present analysis, it was assumed that the vibratory stresses obtained from the present forced response analysis are in a low strain high cycle fatigue $(\mathrm{HCF})$ regime where the nominal strains are elastic without any plastic deformation. We also did not consider potential factors such as stress concentration, temperature, residual stress, and so forth, which could affect the safety margin assessment. Thus, a Goodman diagram approach to predict the safety margins was employed to compensate for the uncertainty of such environmental effects, materials, and production manufacturing, among others. It was also assumed that the static mean stress be accurately predicted from the present numerical methods. Material supplier provided a Goodman limit (i.e. fatigue limit) on their blade material. Hence, blade safety margins were solely predicted over their material fatigue limit given.

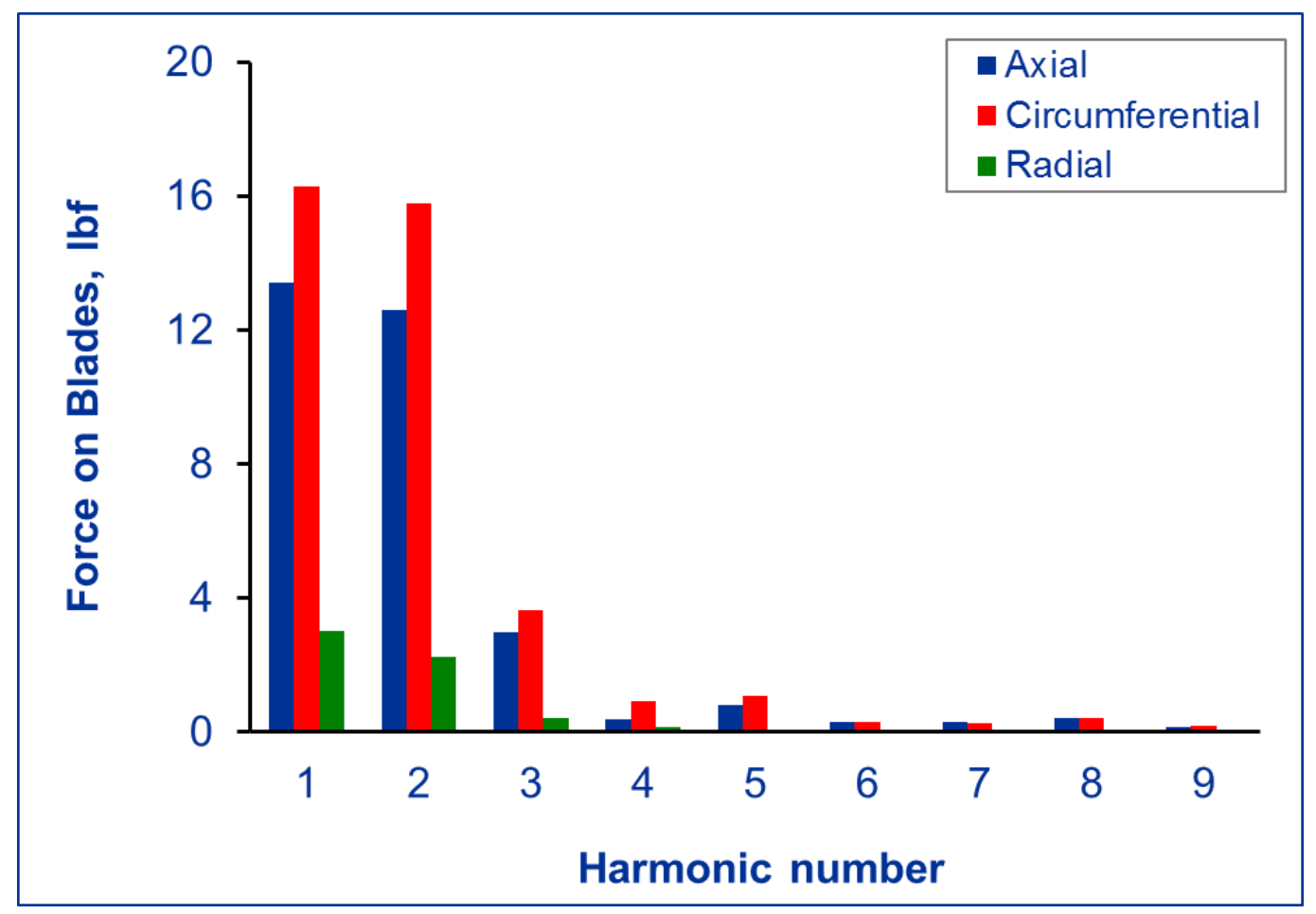

Figure 25: DTF blade unsteady pressure load in terms of EO's (harmonic numbers) at design speed 
Furthermore, additional forced response analyses for the remaining engine order excitations were also carried out repeatedly by applying its individual unsteady aerodynamic excitation load as depicted in Figure 25. Figure 25 shows the contributions of unsteady aerodynamic pressure loads in terms of different EO numbers from Turbo aerodynamic analysis at design speed. It was indicated that the unsteady pressure loads of first three EOs were very significant in amplitude. Relatively small unsteady aerodynamic loads were calculated in higher EOs which were presumably less significant, consequently the inclusion of the high EO frequency responses was not seriously affecting the final dynamic stress values.

A Goodman diagram represented in Figure 26 was constructed to evaluate the safety margins using the sum of EO responses at design speed which displays stress values at every node of the finite element nodal locations in the blade model where they actually occur. Alternating stresses from different EO excitations were superposed at each location in the blade. A simple worst-case stress superposition approach provided a more conservative estimate of the stress state on the life assessment. The fundamental interest from the present cyclic symmetry forced response analysis modeling effort was to determine whether the collective stress state computed at any location in the blade model would lead to fatigue life problem in the currently proposed DTF fan blade configuration. Note that because of the proprietary material limit information, an alternate material limit curve is given in this figure merely for an illustration purpose.

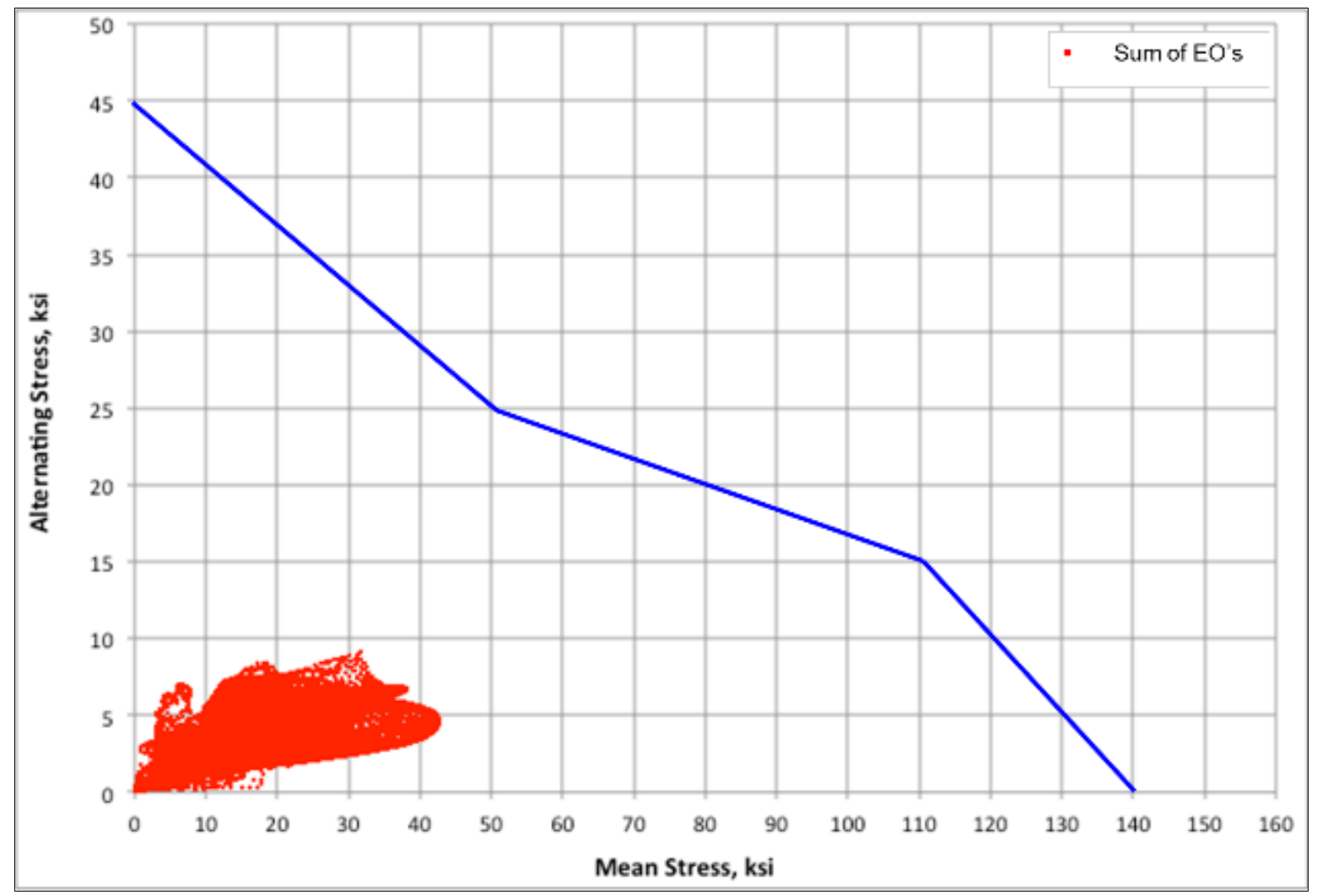

Figure 26: Static and dynamic stresses calculated: sum of EO excitation responses at design speed

As indicated from the present fatigue life assessment based on the stress amplitudes predicted by the present analysis illustrated in Fig. 26, it was concluded that the safety margin requirement set in the current DTF fan blade design was met with a sufficient margin with respect to the operating speed range.

\section{Conclusion and remarks}

The present paper describes a cyclic symmetry finite element analysis modeling technique applied to the forced response analysis of a DTF fan blade subjected to unsteady aerodynamic excitation load resulting from the distorted inlet flow in the fan blade. Only a bladed disk sector was modeled for finite element analysis rather than modeling an entire bladed rotor. This reduction modeling method allows computationally very efficient analysis resulting from a large savings including computational time. This also allows to perform the analysis with adequately fine meshes 
using only a sector model. This savings result from the transformed equations in computations uncoupled, which reduces the order of the equations that must be solved simultaneously roughly to [1/(total number of the sectors)] times the order of the full system. Since this reduction is applied to a single segment prior to the cyclic symmetry transformations, it can greatly reduce the amount of subsequent calculations. This feature is very beneficial especially where typical single blade analysis models are incapable of representing the model for both understanding the harmonic modal blade dynamical behaviors and accurately predicting corresponding forced response information of the blades subjected to complex distorted flow dynamic loading and vibrations.

The analysis result computed from the present modeling technique was compared with the experimental test data. Calculated modal frequencies and unsteady dynamic stresses are in good agreement with the wind tunnel experimental test measurement. Thus, our analysis modeling techniques are fully demonstrated. While the experimental measurement varies to some degree, based on the current analyses, it was concluded that the safety margin requirement set in the DTF fan blade design for the fatigue life was met with a sufficient margin with respect to the operating speed range.

Having validated the present BLI ${ }^{2} \mathrm{DTF}$ fan analysis, result of this cyclic symmetry finite element forced response analysis modeling effort is a very substantial progress in the development of the high-fidelity predictive capability in the forced response analysis subjected to distorted in-flow aerodynamic conditions. This capability enables to support follow-on efforts in BLI propulsion to a variety of applications including development of advanced aircraft and space launch turbomachinery engines being pursued by NASA and industry partners.

Some remarks are addressed for future efforts as follows. While a comparison between analysis results and experimental wind tunnel test results displays a good agreement, yet some differences in dynamic stress magnitudes were observed as shown in Section V.C that should be understood more clearly if more precise assessment is required. In this regard, additional verifications with more cases of the crossing conditions at other off-design speeds, for instance a condition of 2EO Mode 1 are possible. Also, one of the areas to be further assessed would be in vibration damping area. It is very important to gather reliable vibration damping information from true running conditions to correlate the amplitudes of blade response for different modes at different speeds in good assurance, combining the importance of replication of measurements as accurate as possible, which can potentially lead to inaccurate conclusion in computational modeling effort. Respectively from a modeling side, development of the nonlinear damping analysis methods would be considered in support of the further verification process. Similarly, forced response predictions can be improved by a more well-defined fan blade unsteady aerodynamic load through a true integrated inlet-fan unsteady aerodynamic analysis as close as possible to the actual wind tunnel experimental test settings to possibly make further assessments and accuracy for absolute insurance of the forced response analysis results presented in the current analysis. Another area of the importance between analysis and experimental test results may be the presence of blades mistuning. Current analysis assumed that all the blades are identical. If the blade design is based on the assumption of a tuned blade row in which blade rows are identical blades, identically mounted, and uniformly spaced on the disk within a given blade row that ignores the localization of mode shapes, a corresponding difference may exist because of potential differences in its structural and material properties in individual blades. It may be essential to understand the effect of blades mistuning in the designed bladed rotor system where a more accurate assessment on its stress values and fatigue life understanding such effects is required.

Additional remark would be to the use of different materials such as carbon fiber epoxy resin matrix composite or advanced metal materials to improve DTF fan blade performance in distorted flow environments. Use of different material systems may need to be investigated to determine which material would be best suited for maximizing the DTF fan blade structural performance together with weight savings, damping improvement, and operation dependent material properties in $B L I^{2} \mathrm{DTF}$ conditions.

\section{Acknowledgments}

Support of this work by NASA ARMD's Advanced Air Transport Technology Project (Dr. James Heidmann, Project Manager and Mr. Christopher Hughes, Sub-Project Manager) is greatly appreciated. The authors also wish to acknowledge to thank Mr. David Arend of NASA GRC for his leadership of the BLI²DTF task.

\section{References}

[1] L. W. Hardin, G. Tillman, O. P. Sharma, J. Berton, D. J. Arend: Aircraft System Study of Boundary Layer Ingesting Propulsion, 48th AIAA/ASME/SAE/ASEE Joint Propulsion Conference, 2012, Atlanta, Georgia.

[2] A.C. Huang, D.K. Hall, A. Uranga, E. Greitzer: Power Balance Assessment of BLI Benefits for Civil Aircraft, AIAA Science and Technology Forum and Exposition, 2015. 
[3] Yehia El-Aini, Robert deLaneuville, Alan Stoner, Vincent Capece: High cycle fatigue of turbomachinery components - Industry perspective, AIAA Paper No. 97-3365, 33rd Joint Propulsion Conference and Exhibit, 1997. [4] Arend, D. J., Wolter, J. D., Hirt, S. M., Provenza, A. J., Gazzaniga, J. A., Cousins, W. T., Hardin, L. W., Sharma, O. P.: Experimental Evaluation of an Embedded Boundary Layer Ingesting Propulsor for Highly Efficient Subsonic Cruise Aircraft, AIAA Paper 2017-5041, July 2017.

[5] Cousins, W.T., Voytovych, D.M., Tillman, T.G., Gray, E.: Design of a Distortion-Tolerant Fan for a BoundaryLayer Ingesting Embedded Engine Application, AIAA Paper 2017-5042, AIAA Propulsion and Energy Forum, Atlanta, GA, July 10-12, 2017.

[6] ANSYS finite element analysis code, Canonsburg, Pennsylvania, USA.

[7] Dickens, John M.: Numerical Methods for Dynamic Substructure Analysis, Ph.D. Thesis, University of California, Berkeley, 1980.

[8] E. Gray: private communication on finite element mesh and BLI ${ }^{2}$ DTF blade geometry, United Technologies Corporation United Technologies Research Center.

[9] J.C. Simo, T.A. Laursen: An Augmented Lagrangian Treatment of Contact Problems Involving Friction, Computers and Structures. Vol. 42, No. 1.97-116. 1992.

[10] M. A. Bakhle, T. S. R. Reddy, R. M. Coroneos, J. B. Min, A. J. Provenza, K. P. Duffy, G. L. Stefko, G. S. Heinlein: Aeromechanics Analysis of a Distortion-Tolerant Fan with Boundary Layer Ingestion, AIAA SciTech Forum, January 2018 (submitted for publication). CFD analysis and references therein for the TURBO code.

[11] W. Carnegie: Vibrations of Rotating Cantilever Blading, J. of Mechanical Eng. Science, Vol. 1, No. 3, 1959. [12] J.A. Stricklin, W.E. Haisler, W.A. von Risemann: Evaluation of Solution Procedures for Material and/or Geometrically Nonlinear Structural Analysis, AIAA J. Vol. 11, 1973, pp. 292-299.

[13] C. Rajakumar, C.R. Rogers: The Lanczos Algorithm Applied to Unsymmetric Generalized Eigenvalue Problems, International Journal for Numerical Method in Engineering. Vol. 32. 1009-1026. 1991.

[14] Klaus-Jurgen Bathe: Finite Element Procedures in Engineering Analysis, Prentice-Hall, 1982.

[15] Arthur P. Boresi, Omar M. Sidebottom: Advanced Mechanics of Materials, John Wiley \& Sons, 1985.

[16] Provenza, A. J., Duffy, K. P., Bakhle, M. A.: Aeromechanical Response of a 22" Fan Operating in a Heavily Distorted Axial Flow, ASME Turbo Expo, June 2018 (submitted for publication).

[17] K. P. Duffy, A. J. Provenza, M. A. Bakhle, J. B. Min, A. Abdul-Aziz: Laser Displacement Measurements of Fan Blades in Resonance and Flutter During the Boundary Layer Ingesting Inlet - Distortion -Tolerant Fan Wind Tunnel Test, AIAA SciTech Forum, January 2018 (submitted for publication). 